\title{
Constrained interest rates and changing dynamics at the zero lower bound
}

\section{Journal Article}

Author(s):

Bäurle, Gregor; Kaufmann, Daniel (D; Kaufmann, Sylvia; Strachan, Rodney

Publication date:

2020-04

Permanent link:

https://doi.org/10.3929/ethz-b-000410278

Rights / license:

In Copyright - Non-Commercial Use Permitted

Originally published in:

Studies in Nonlinear Dynamics \& Econometrics 24(2), https://doi.org/10.1515/snde-2017-0098 


\title{
Gregor Bäurle' / Daniel Kaufmann² / Sylvia Kaufmann³ / Rodney Strachan ${ }^{4}$
}

\section{Constrained interest rates and changing dynamics at the zero lower bound}

\author{
${ }^{1}$ Swiss National Bank, Zurich, Switzerland, E-mail: gregor.baeurle@snb.ch \\ ${ }^{2}$ Université de Neuchâtel, Institut de Recherches Économiques, Neuchâtel and KOF Swiss Economic Institute, ETH Zurich , \\ Switzerland. https://orcid.org/0000-0003-3260-0660. \\ ${ }^{3}$ Study Center Gerzensee, Gerzensee, Switzerland, E-mail: sylvia.kaufmann@szgerzensee.ch \\ 4 University of Queensland, School of Economics, Brisbane, Australia, E-mail: r.strachan@uq.edu.au
}

\begin{abstract}
:
The interaction of macroeconomic variables may change as nominal short-term interest rates approach zero. In this paper, we propose to capture these changing dynamics with a state-switching parameter model which explicitly takes into account that the interest rate might be constrained near the zero lower bound by using a Tobit model. The probability of state transitions is affected by the lagged level of the interest rate. The endogenous specification of the state indicator permits dynamic conditional forecasts of the state and the system variables. We use Bayesian methods to estimate the model and to derive the forecast densities. In an application to Swiss data, we evaluate state-dependent impulse-responses to a risk premium shock identified with sign-restrictions. We provide an estimate of the latent rate, i.e. the rate lower than the constraint on the interest rate level which would be state- and model-consistent. Additionally, we discuss scenario-based forecasts and evaluate the probability of exiting the ZLB region. In terms of log predictive scores and the Bayesian information criterion, the model outperforms a model substituting switching with stochastic volatility and another including intercept switching only combined with stochastic volatility.
\end{abstract}

Keywords: constrained variable, regime switching, stochastic volatility, time-varying probability, Tobit model JEL classification: C3, E3

DOI: $10.1515 /$ snde-2017-0098

\section{Introduction}

The monetary policy instrument of leading central banks is a nominal short-term interest rate. Until recently, the use of this instrument was perceived to face one problem, however. Because moderate amounts of money can be stored at relatively low cost, the effective nominal interest rate cannot fall below or not far below zero. Some discussion on the zero lower bound (ZLB) took place in the beginning of the century (Auerbach and Obstfeld, 2005; Benhabib, Schmitt-Grohe and Uribe, 2002; Eggertsson and Woodford, 2003; Eggertsson et al., 2004; Woodford, 2003) and it was recognized that the ZLB might fundamentally change the functioning of an economy. Nevertheless, at the time the ZLB was not perceived to constitute a major problem Reifschneider and Williams (2000). However, since the outbreak of the financial crisis and the following euro area sovereign debt crisis, the policy rate has remained for a considerably long period of time at the ZLB, particularly in the US but also in Switzerland. To circumvent the constraint of the ZLB on the policy rate, the US resorted to unconventional monetary policy measures to accommodate the negative effects of the financial crash on the real economy. In Switzerland, the Swiss National Bank (SNB) responded to deflationary appreciation pressures by intervening in the foreign exchange market, by buying corporate bonds in 2009/2010 and by introducing a minimum exchange rate against the euro in September 2011. In December 2014, the SNB announced that it was lowering the range for its operational target, i.e. for the three-month libor, into negative territory to between $-0.75 \%$ and $-0.25 \%$. In January 2015 , it discontinued the euro-Swiss franc exchange rate floor and moved the target range further into negative territory to between $-1.25 \%$ and $-0.25 \%$.

While negative policy rates are now being implemented, under the current monetary arrangements there is still an effective lower bound at which they are constrained. As long as cash currency is available, there will be a lower bound at which it will pay agents to incur the storage costs and substitute a deposit account with cash. Moreover, if economic dynamics are changing near this effective ZLB, it is also an open issue whether shocks or interest rate changes have the same effect on, for example, prices, exchange rates and GDP, as when interest rates are out of the ZLB region. One reason for this is that usually, and obviously, the ZLB region is reached 
because of strongly deteriorating economic and financial conditions. In these periods, uncertainty rises which may change the interest rate sensitivity of economic agents. Moreover, adverse shocks may have different effects if agents expect that the central bank has limited ability to counteract those shocks with further interest rate cuts.

In this paper, we base the analysis on a vector autoregression (VAR) with parameters that are allowed to change when the ZLB becomes binding. A latent state indicator determines the state-specific parameters and the error covariances of the VAR system. The probability distribution of the state indicator depends itself on a covariate which is perceived to be informative on the prevailing state. A natural candidate is the interest rate. Furthermore, we take into account that the interest rate might be constrained by working with a Tobit approach. We are able to provide an estimate of the latent interest rate, i.e. the rate below the constrained one which would be state- and model-consistent. Our model therefore provides an intuitive measure of whether the ZLB is a binding constraint. The method can be adapted to situations in which several variables are constrained permanently or temporarily. This becomes important in situations in which unconventional monetary policy targets directly prices in specific asset markets, e.g. government bonds, mortgage or currency markets.

Our research relates to a growing empirical literature that studies macroeconomic dynamics at the ZLB using structural VARs. ${ }^{1}$ Studies relying on a constant parameter VAR include Miyao (2002) and Schenkelberg and Watzka (2013). Closer to our approach is Iwata and $\mathrm{Wu}$ (2006), who look at the Japanese experience with a constant-parameter structural VAR, but take into account that the interest rate is a constrained variable. A few papers allow for changing parameters at the ZLB. Baumeister and Benati (2013) explore how a compression in the bond spreads impact the economy during the Great Recession using a time-varying parameter VAR estimated for the US, the Euro area, Japan and the UK. Debortoli, Galí, and Gambetti (2018) use a time-varying parameter VAR to examine whether nonconventional policy actions are effective substitutes for traditional interest rate cuts. Nakajima (2011) extends the time-varying parameter VAR by modelling the interest rate as a censored variable and estimates the model with Japanese data. Kimura and Nakajima (2016) analyze Japan's monetary policy using a time-varying parameter VAR with a specific focus on the identification of, and switching between, unconventional and conventional monetary policy shocks. Wu and Xia (2016) assess how parameters of a VAR changed when the interest rate reached the ZLB in the US relying on a latent interest rate derived from a term-structure model. Similarly, Bäurle and Kaufmann (2018) study how the response of Swiss macroeconomic aggregates to risk premium shocks is affected by the ZLB. None of these contributions models the endogenous change of parameters when the interest rates approaches the ZLB. This is, however, an essential feature especially if the focus is on forecasting. Furthermore, an endogenous change in parameters can be motivated on the basis of non-linear dynamic stochastic general equilibrium models in which the ZLB is treated as an occasionally binding constraint as in Gust et al. (2017) or Plante, Richter, and Throckmorton (2018).

We apply our method to analyze the dynamics of Swiss data, namely GDP, the consumer price index (CPI), the effective exchange rate in relation to the nominal interest rate. Taking up the idea of Bäurle and Kaufmann (2018), we analyze how risk premium shocks affecting the exchange rate transmit to prices. We find that risk premium shocks have more persistent effects on prices if the policy rate is constrained, but have only temporary effects if rates can accommodate. The endogenous specification of the state indicator allows us to compute dynamic state and variable forecasts. We provide scenario-based forecasts over the period 2014, third quarter, to 2020, third quarter. We find that the system is unlikely to exit the ZLB region as long as appreciation pressures are present. In terms of log predictive score and the Bayesian information criterion, the model performs better than an alternative model substituting switching volatility with stochastic volatility and another which combines switching intercepts, time-invariant autoregressive parameters and stochastic volatility.

The next section presents the econometric model and discusses various aspects of the endogenous state probability distribution. Section 3 presents the estimation procedure and describes the computation of the unconditional and the scenario-based forecasts. The results are discussed in Section 4 . Section 5 compares the performance of the model against a model substituting switching volatility with stochastic volatility and another one which combines switching intercepts, time-invariant autoregressive parameters and stochastic volatility. Section 6 concludes. The interested reader may find technical details about the distributional properties of censored and uncensored variables in Appendix A. The Bayesian framework including prior specifications and the derivation of posterior distributions is outlined in Appendix B. Appendix C displays additional results of the various estimated models. 


\section{Econometric model}

\subsection{Specification}

To investigate changing dynamics among $N$ variables $y_{t}$, we frame the model within a time-varying parameter VAR of lag order $p$

$$
y_{t}=\mu_{t}+B_{1 t} y_{t-1}+\cdots+B_{p t} y_{t-p}+\varepsilon_{t}, \varepsilon_{t} \sim N\left(0, \Sigma_{t}\right)
$$

which is compactly written in regression form:

$$
y_{t}=X_{t} \beta_{t}+\varepsilon_{t}, \varepsilon_{t} \sim N\left(0, \Sigma_{t}\right)
$$

where $X_{t}=I_{N} \otimes\left[1, y_{t-1}^{\prime}, \ldots, y_{t-p}^{\prime}\right]$ and $\beta_{t}=\operatorname{vec}\left(\left[\mu_{t}, B_{1 t}, \ldots, B_{p t}\right]^{\prime}\right)$. The regression reflects explicitly that we condition on past observed values $y_{t-1}, \ldots, y_{t-p}$, which includes interest rates at their low level as observed recently. To capture the notion that conditional on past observed values the changing dynamics may have implied lower interest rates than actually observed, or in other words that central banks felt constrained by the zero lower bound, we extend the regression and write

$$
\begin{aligned}
& y_{t}^{*}=X_{t} \beta_{t}+\varepsilon_{t} \\
& \tilde{y}_{1 t}=\max \left\{y_{1 t}^{*}=y_{1 t}, b\right\} \\
& \tilde{y}_{2 t}=y_{2 t}^{*}=y_{2 t}
\end{aligned}
$$

We construct $\tilde{y}_{t}$ from the originally observed data. The sub-vector $\tilde{y}_{1 t}$ collects the variables we perceive as potentially constrained. Period- $t$ values equal $y_{1 t}$ if $y_{1 t}$ is higher than $b$, and are censored at $b$ if $y_{1 t}$ is lower than $b$. All variables perceived as unconstrained are collected in $\tilde{y}_{2 t}$ and all values in $\tilde{y}_{2 t}$ equal the observed values $y_{2 t}$. In our application, $y_{1 t}$ is the nominal interest rate and re-defining it yields a variable censored at $b .^{2}$ However, contrary to standard censored data, the lower bound $b$ is not pre-determined by our data. The interest rate is always observed and we have to choose $b$. We interpret $b$ as the level of interest rates at which central banks became constrained in setting the policy rate, forcing them to implement unconventional policy measures. Thus, the relevant threshold $b$ is not necessarily equal to zero, but might be at a value above, but close to zero. In the application we set $b=0.25$, defining periods of constrained interest rates as those when the policy rate decreased below 25 basis points (bps). In the empirical section we provide additional justification for setting $b=0.25 .^{3}$

We might also envisage to use a model in which the interest rate has truncated support with a moving lower truncation threshold given e.g. by the lower bound of the libor target in the SNB's case. We do not pursue this avenue, because we also want to evaluate the extent to which policy is constrained, conditional on all available observed values. If we condition on periods in which we assume the central bank has been constrained, the Tobit framework allows us to form a model-based estimate of the latent interest rate $y_{1 t}^{*}$, i.e. the interest rate level which would be consistent with the model and current and past observed data.

To model the time-varying process of the parameters, we rely on a mixture approach

$$
\begin{aligned}
& \beta_{t}=\beta_{0}\left(1-I_{t}\right)+\beta_{1} I_{t} \\
& \Sigma_{t}=\Sigma_{0}\left(1-I_{t}\right)+\Sigma_{1} I_{t}
\end{aligned}
$$

where the latent indicator $I_{t} \in\{0,1\}$ drives the changing coefficients and the error covariance of the system. We define $I_{t}=1$ to indicate periods in which the interest rate is close to the ZLB, i.e. is close to being constrained. We call these periods the critical ZLB region. Coefficients and the error variance are allowed to change, given that the volatility of constrained variables may change and their covariance with other variables as well. The indicator $I_{t}$ may be specified ad hoc by defining a priori the periods of very low interest rates. The disadvantage of this procedure is that the relevant threshold for the interest rate at which dynamics change is in fact unknown to the investigator.

Therefore, we set up a probabilistic model for $I_{t}$ which allows us to estimate it conditional on the data. A natural indicator of whether $I_{t}$ turns out to be 0 or 1 is the deviation of the lagged interest rate from the ZLB, $r_{t-1}$. To be consistent with model setup (1), we condition again on past observed values:

$$
p_{1 t}=P\left(I_{t}=1 \mid r_{t-1}, \gamma, \gamma^{r}\right)=\frac{\exp \left(\gamma^{r} r_{t-1}+\gamma\right)}{1+\exp \left(\gamma^{r} r_{t-1}+\gamma\right)}
$$


Model (1)-(3) differs from a smooth transition VAR specification where variables would be explained by a mixture of two states (Teräsvirta and Anderson, 1992; Auerbach and Gorodnichenko, 2012):

$$
y_{t}^{*}=X_{t} \beta_{0}\left(1-p_{1 t}\right)+X_{t} \beta_{1} p_{1 t}+\varepsilon_{t}, \varepsilon_{t} \sim N\left(0, \Sigma_{0}\left(1-p_{1 t}\right)+\Sigma_{1} p_{1 t}\right)
$$

By taking on either value $\{0,1\}$, the state indicator $I_{t}$ in (2) determines which dynamic regime characterizes period $t$-specific variables. Posterior inference on $I_{t}$ will indicate how well both periods can be discriminated, i.e. whether a regime-switching representation is to be preferred over a smooth transition VAR representation.

\subsection{Some considerations on the probability function}

To identify $I_{t}=1$ in (3) as corresponding to periods in which the interest rate is in the critical ZLB region, we restrict $\gamma^{r}<0$. Moreover, we call parametrization (3) the implicit threshold parametrization because we are able to recover the threshold interest rate after having estimated the model. The threshold is defined as the level of $r_{t}$ at which the state probability equals 0.5 . For example, if the interest rate were expressed in percentage terms and if $\gamma^{r}=-1$ and $\gamma=0.5$, then the threshold level would lie at $-\gamma / \gamma^{r}=0.5 \%$. To estimate the model, we use parametrization (3) because the non-linear model for the transition probabilities becomes linear in $\gamma^{r}$ and $\gamma$ after two layers of data augmentation. This allows us to draw from full conditional distributions.

The usual parametrization, which we call the explicit threshold parametrization, includes explicitly the threshold $\tilde{\gamma}$ :

$$
P\left(I_{t}=1 \mid r_{t-1}, \gamma, \gamma^{r}\right)=\frac{\exp \left(\gamma^{r}\left(r_{t-1}-\tilde{\gamma}\right)\right)}{1+\exp \left(\gamma^{r}\left(r_{t-1}-\tilde{\gamma}\right)\right)}
$$

Note that from an estimate of (3), in case $\gamma^{r} \neq 0$, we can also retrieve the threshold level $\tilde{\gamma}$,

$$
\begin{aligned}
-\gamma^{r} \tilde{\gamma} & =\gamma \\
\tilde{\gamma} & =-\gamma / \gamma^{r}
\end{aligned}
$$

Relation (5) indicates that the threshold $\tilde{\gamma}$ and $\gamma$ are mutually highly dependent. Conditional on $\gamma^{r}$, the threshold determines $\gamma$ and vice versa. For a given threshold, $\gamma$ is increasing in $\gamma^{r}$. Figure 1 illustrates this point. The figure plots values for the short term interest rate against the state probability obtained for various $\gamma^{r}$, assuming a threshold level of $0.8 \%$. As $-\gamma^{r}$ increases, the probability function approaches a step function. Conditional on the threshold, $\gamma$ is increasing proportionally to $\gamma^{r}$.

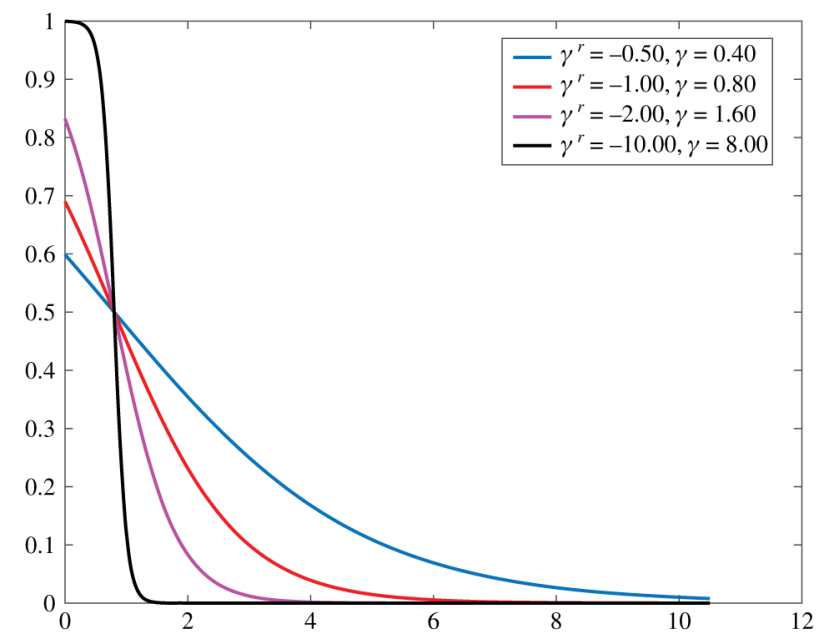

Figure 1: State probability $P\left(I_{t}=1 \mid r_{t-1}, \gamma^{r}, \gamma\right)$ for various sensitivities $\gamma^{r}$, where $\gamma$ is adjusted to keep the threshold level at $0.8 \%$.

The relationship between both parameterizations can be used to include information into the prior distribution for the parameters of the state probabilities. We may have some idea of an upper and of a lower bound for $\widetilde{\gamma}$. For example, $\widetilde{\gamma}$ is certainly well below $10 \%$, is probably below $1 \%$, and perhaps between $0.5 \%$ and $1.5 \%$. So, let the upper and lower bound on $\widetilde{\gamma}$ be $\bar{\gamma}$ and $\underline{\gamma}$, respectively, such that $\underline{\gamma} \leq \tilde{\gamma} \leq \bar{\gamma}$. 
This implies $\underline{\gamma} \leq-\frac{\gamma}{\gamma^{r}} \leq \bar{\gamma}$, or

$$
-\gamma^{r} \underline{\gamma} \leq \gamma \leq-\gamma^{r} \bar{\gamma}
$$

This puts an upper and a lower bound on $\gamma$ since $\gamma^{r}<0$. The prior for $\left(\gamma, \gamma^{r}\right)$ is expressed with these inequalities in place:

$$
\pi\left(\gamma, \gamma^{r}\right)=N\left(g_{0}, G_{0}\right) 1\left(\gamma^{r}<0\right) 1\left(-\gamma^{r} \underline{\gamma} \leq \gamma \leq-\gamma^{r} \bar{\gamma}\right)
$$

We may also work with parametrization (4). Various priors have been suggested in the literature, see Lopes and Salazar (2005) for an overview. Using this parametrization, a truncated normal prior distribution may be used to incorporate prior information on the threshold.

\section{Estimation and forecasting}

\subsection{Estimation}

The estimation of model (1)-(3) proceeds along the lines of Chib (1992). We use data augmentation to obtain posterior inference on the model parameters in (1). The augmented data will be an estimate of the underlying values $y_{1 t}^{*}$ of the constrained observations in $\tilde{y}_{1 t}$. In the following, bold faced objects gather all observations of a vector, e.g. $\boldsymbol{y}=\left\{y_{t} \mid t=1, \ldots, T\right\}$ or $\mathbf{X}=\left\{X_{t} \mid t=1, \ldots, T\right\}$, and similarly for $\boldsymbol{y}^{*}$ and $\mathbf{I}$. We gather the latent values underlying the censored observations in $y_{1}^{*}=\left\{y_{1 t}^{*} \mid t \in \boldsymbol{t}^{*}\right\}, \boldsymbol{t}^{*}=\left\{\tau \mid y_{1 \tau} \leq b, \tau=1, \ldots, T\right\}$ while $y_{2}^{*}$ contains all uncensored observations, $\boldsymbol{y}_{\mathbf{2}}^{*}=\left\{\boldsymbol{y}^{*} \backslash \boldsymbol{y}_{\mathbf{1}}^{*}\right\}$. The parameters are included in $\theta=\left\{\beta_{k}, \Sigma_{k}, \boldsymbol{\gamma} \mid k=0,1, \boldsymbol{\gamma}=\left(\gamma^{r}, \gamma\right)\right\}$, and the augmented parameter vector contains the latent variables in addition to $\theta, \vartheta=\left\{\theta, \boldsymbol{y}_{1}^{*}, \mathbf{I}\right\}$.

Data augmentation allows us to express the posterior distribution in terms of the complete data likelihood and to design a Bayesian Markov chain Monte Carlo (MCMC) scheme to estimate the model:

$$
\pi(\vartheta \mid \tilde{y}) \propto f\left(\mathbf{y}^{*} \mid \mathbf{X}, \mathbf{I}, \theta\right) \pi(\mathbf{I} \mid \mathbf{r}, \boldsymbol{\gamma}) \pi\left(\mathbf{y}_{\mathbf{1}}^{*}\right) \pi(\theta)
$$

To sample from (8), we draw iteratively from the conditional posterior of

i. $\mathbf{I}, \pi\left(\mathbf{I} \mid y^{*}, \mathbf{X}, \mathbf{r}, \theta\right)$

ii. $y_{1}^{*}, \pi\left(y_{1}^{*} \mid y_{2}^{*}, \mathbf{X}, \mathbf{I}, \theta\right) 1\left(y_{1}^{*} \leq b\right)$

iii. $\boldsymbol{\gamma}, \pi(\boldsymbol{\gamma} \mid \mathbf{r}, \mathbf{I}) 1\left(\gamma^{r}<0\right) 1\left(-\gamma^{r} \underline{\gamma} \leq \gamma \leq-\gamma^{r} \bar{\gamma}\right)$

iv. the parameters, $\pi\left(\operatorname{vec}\left(\left[\beta_{0}, \beta_{1}\right]\right) \mid \boldsymbol{y}^{*}, \mathbf{X}, \mathbf{I}, \Sigma_{0}, \Sigma_{1}\right), \pi\left(\Sigma_{k} \mid \boldsymbol{y}^{*}, \mathbf{X}, \mathbf{I}, \beta_{k}\right), k=0,1$.

All posterior distributions are standard distributions. Given that there is no state persistence, in step (i) we can sample $\mathbf{I}$ in one draw from a discrete distribution. Conditional on observed values $\boldsymbol{y}_{2}^{*}$ and $\mathbf{X}$, the state $\mathbf{I}$ and the model parameters, we draw $\boldsymbol{y}_{1}^{*}$ from a truncated normal distribution. To derive the posterior of the parameters governing the state distribution, we condition on two layers of data augmentation (see FrühwirthSchnatter and Frühwirth, 2010; Kaufmann, 2015). In the first layer, we obtain a linear model with non-normal error terms, which relates the difference in latent state utilities to the interest rate effect on the state probability. In a second layer, we approximate the exponential error distribution by a mixture of $M$ normals. Conditional on the differences in latent utilities and the components of the mixtures, the posterior of $\gamma$ is normal. We draw from the normal posterior truncated to the region where the parameters restriction derived in (7) are fulfilled. The posterior distribution of the remaining parameters $\beta_{k}$ and $\Sigma_{k}, k=0,1$, in (iv) are normal and inverse Wishart, respectively. The interested reader finds a detailed derivation of distributional data properties and the Bayesian approach including prior specifications and the derivation of posterior distributions in Appendices A and B, respectively. 


\subsection{Conditional forecasting}

The model can be used to obtain forecasts over the forecast horizon $H, h=1, \ldots, H$. To obtain draws from the unconditional posterior predictive distribution at each horizon $h$ :

$$
\pi\left(y_{T+h} \mid y_{T}\right) \propto \prod_{j=1}^{h} \pi\left(y_{T+j} \mid y_{T+j-1}, I_{T+j}\right) \pi\left(I_{T+j} \mid y_{T+j-1}\right)
$$

we produce dynamic forecasts and simulate for $j=1, \ldots, h$, we draw

1. $I_{T+j}^{(l)}$ from $\pi\left(I_{T+j}^{(l)} \mid r_{T+j-1}^{(l)}, \gamma^{(l)}\right)$, with $r_{T}^{(l)}=r_{T}$, and then

2. $y_{T+j}^{(l)}$ from $\pi\left(y_{T+j}^{(l)} \mid y_{T+j-1}^{(l)}, I_{T+j}^{(l)}, \theta^{(l)}\right) \sim N\left(m_{T+j}^{(l)}, \Sigma_{I_{T+j}^{(l)}}\right)$

with $m_{T+j}^{(l)}=X_{T+j}^{(l)} \beta_{I_{T+j}^{(l)}} y_{T}^{(l)}=y_{T}$ and $X_{T+1}^{(l)}=X_{T+1}$.

for each draw $(l)$ out of the posterior $\pi(\vartheta \mid \tilde{y})$.

We may also produce so-called conditional forecasts, which would reflect specific scenarios. In all examples, step 2 above is adjusted appropriately. For example

2. (i) keep the mean forecast of the interest rate at or above the last rate, i.e. restrict the predictive distribution to:

$$
\pi\left(y_{T+h} \mid y_{T},\left(m_{1, T+1}, \ldots, m_{1, T+h}\right)=y_{1 T}\right) \text { or } \pi\left(y_{T+h} \mid y_{T},\left(m_{1, T+1}, \ldots, m_{1, T+h}\right) \geq y_{1 T}\right)
$$

The second conditional forecast is implemented as follows. At each step $j, j=1, \ldots, h$, we set $m_{1, T+j}^{(l)}=$ $\max \left\{X_{1, T+j}^{(l)} \beta_{1, I_{T+j}^{(l)}}, y_{1 T}\right\}$.

2. (ii) implement a (mean) path for a variable $i$ over a certain period of time, say $h=1, \ldots, 4$, (e.g. lower the interest rate to $-1 \%$ for one year):

Simulate first variable $i, y_{i, T+h^{\prime}}^{(l)}$ from $N\left(m_{i, T+h}, \Sigma_{i, I_{T+h}^{(l)}}\right)$, where $m_{i, T+h}$ is pre-specified.

Then, conditional on $y_{i, T+h^{\prime}}^{(l)}$, simulate all other variables, $y_{-i, T+h}^{(l)} \mid y_{i, T+h^{\prime}}^{(l)}$ from $N\left(m_{-i \mid i, T+h}, \Sigma_{-i \mid i, I_{T+h}}\right)$, the moments of which are given by the moments of the implied normal conditional predictive distribution.

2. (iii) a combination of the two. Here we apply 2. (ii) in combination with 2. (i) for the mean forecast, i.e. $m_{i, T+h}^{(l)}=m_{i, T+h}$ for $h=1, \ldots 4$, and $m_{i, T+h}^{(l)}=y_{i, T+h-1}^{(l)}$ for $h=5, \ldots, H .{ }^{4}$

\section{Results}

\subsection{Data and prior specification}

To illustrate the method, we estimate a level VAR for four Swiss variables, namely GDP, the CPI, the three-month Swiss franc libor and the trade-weighted effective exchange rate. We use quarterly data covering the period 1974, first quarter, to 2014, third quarter. As already mentioned, the state-identifying restriction $\gamma^{r}<0$ defines $I_{t}=1$ as indicating the periods in which the interest rate enters the ZLB critical region. We additionally induce the threshold, i.e. the level of the interest rate at which $P\left(I_{t}=1\right)=0.5$, to lie in the interval $[\gamma, \bar{\gamma}]=[0.5,1.5]$. Hence the prior mean for the threshold is 1.0. In the ZLB critical region, we define interest rate levels at or below 0.25 as those being constrained, i.e. $b=0.25$. 


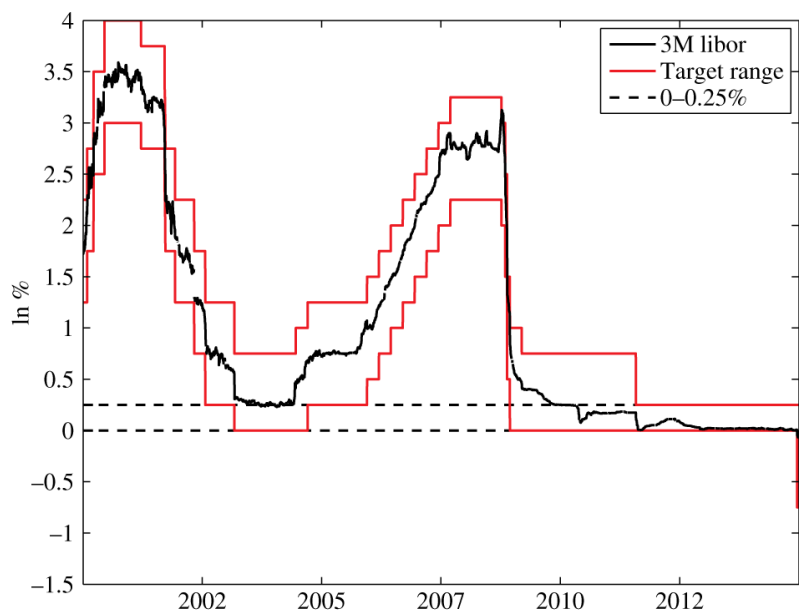

Figure 2: Three-month libor with target range.

This assumption defines the interest rate since 2010 and two observations at the end of the 1970s as being constrained. ${ }^{5}$ Figure 2 shows that when the SNB set the point target for the three-month Swiss franc libor to this value, notably in March 2003 and March 2009, it also narrowed the target range from the usual 100 bps to 75 bps. While in March 2003 the SNB justified the narrowed target range by technical factors without explaining in more details, on 12 March 2009 the SNB communicated that the narrowed target range "is due to the fact that a negative libor is not technically possible" (SNB 2003, 2009). On 19 March 2009, the SNB discussed negative interest on reserves as an additional tool to loosen policy further (Jordan 2009). Although this was an option, the truncated target range suggests that the SNB viewed it impossible to push the libor into negative territory under the current monetary regime. As a consequence, the ZLB was effectively binding because the SNB was not yet willing to resort to this nonconventional policy. It continued to be binding in August 2011 when the SNB narrowed the target range to $25 \mathrm{bps}$ and imposed an exchange rate floor against the euro in September 2011. In December 2014 the target range widened again to $100 \mathrm{bps}$ when the SNB announced to introduce negative interest on reserves, a month before the minimum exchange rate level was discontinued.

This history leads us to interpret that until the end of 2014, the SNB and in fact all economic agents viewed the libor as increasingly constrained by the ZLB whenever it was falling to $0.25 \%{ }^{6}$ This interpretation is qualitatively in line with survey expectations and libor futures contracts. Figure 3 shows expectations from the Swiss Economic Institute (KOF) Consensus Survey, a survey of professional forecasters for Switzerland. ${ }^{7}$ The 3-month forecast (in Panel (a)) dropped virtually to zero in August 2011 when the SNB intoduced the minimum exchange rate level and announced a libor target as close to zero as possible (SNB 2011). No forecaster expected the libor to fall below zero until 2015. The 12-month ahead forecasts (in Panel (b)) show that, before the introduction of the minimum exchange rate, a fair share of survey participants expected interest rates even to increase within twelve months. However, during the minimum exchange rate regime, interest rate expectations of most participants remained close to zero. ${ }^{8}$

\section{A}

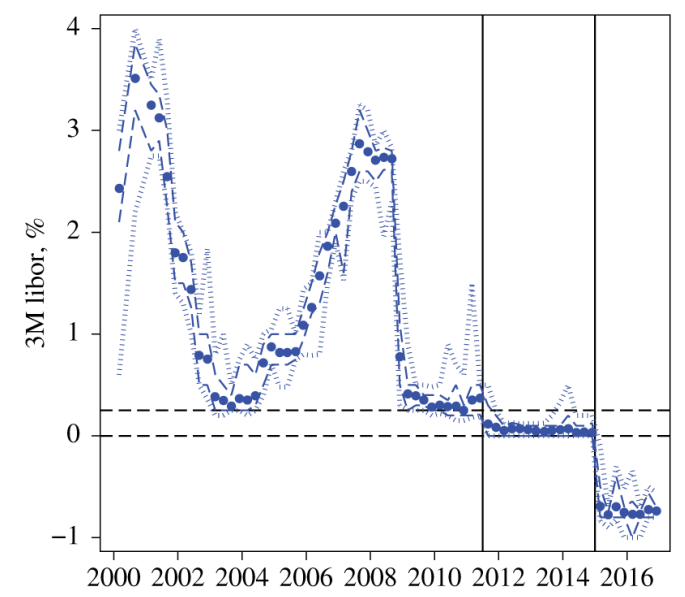

B

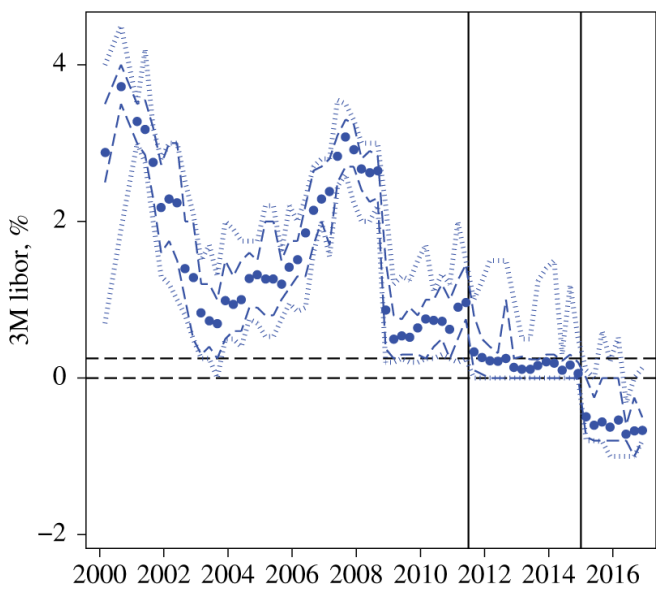

Figure 3: KOF Consensus Survey.

Professional forecasters' libor expectations. Mean forecast (dots), 10th and 90th percentiles (dashed), minimum and maximum (dotted). The dashed horizontal lines give the $0-0.25 \%$ range. The vertical lines indicate the introduction and removal of a minimum exchange rate against the euro. (A) 3-months ahead. (B) 12-months ahead 
For estimation, we adopt the following prior specifications for the model parameters and the latent variable $y_{1}^{*}$, see Appendix B for the specification of hyperparameters:

1. We assume independent Minnesota type prior for the VAR parameters $\beta_{k}, k=0,1, \pi\left(\beta_{k}\right)=N(\underline{v}, \underline{V})$ (Doan, Litterman and Sims, 1984; Bańbura, Giannone and Reichlin, 2010).

2. For $\Sigma_{k}$, we assume inverse Wishart prior distributions $\pi\left(\Sigma_{k}\right) \sim I W\left(\underline{s}, \underline{S}_{k}\right)$, where the scale $\underline{S}_{k}$ is proportional to the variance of residuals of state-specific univariate autoregressions, $\underline{S}_{k, i i} \propto \sigma_{k i}^{2}$, with pre-defined states $\underline{I}_{t}=1$ if the libor $\leq 1 \%$.

3. A relatively informative prior on $\gamma^{r}$ is used to obtain a steep shape of the transition function (see Figure 1), and also because there are only a few observations near the ZLB.

$$
\left(\begin{array}{l}
\gamma^{r} \\
\gamma
\end{array}\right) \sim N\left(\left[\begin{array}{r}
-10 \\
10
\end{array}\right], \operatorname{diag}(0.01,6.25)\right) 1\left(\gamma^{r}<0\right) 1\left(-0.5 \gamma^{r} \leq \gamma \leq-1.5 \gamma^{r}\right)
$$

Figure 4, Panels (a) and (b), plot the prior and posterior distributions of $\gamma^{r}$ and $\gamma$, respectively. Although the prior distribution for $\gamma^{r}$ is quite tight (broken blue line), it is considerably updated conditional on the data (solid blue). We observe a considerable update for $\gamma$, too (broken and solid red). Panel (c) shows what the parameters imply for the transition function. Compared to the $95 \%$ highest prior density interval (left blue), the posterior interval (right red) is quite dense and concentrated at the upper bound pre-specified for the threshold.

4. For $y_{1}^{*}$, we work with a diffuse prior, $\pi\left(y_{1}^{*}\right) \propto 1\left(y_{1}^{*} \leq b\right)$.

A

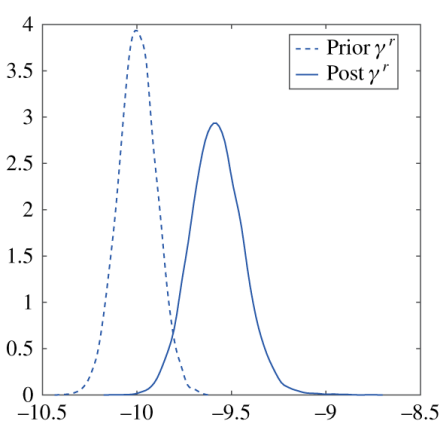

B

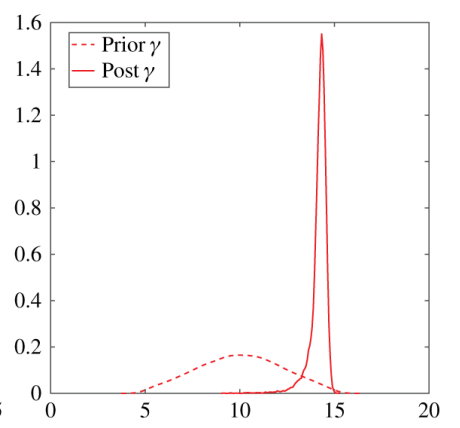

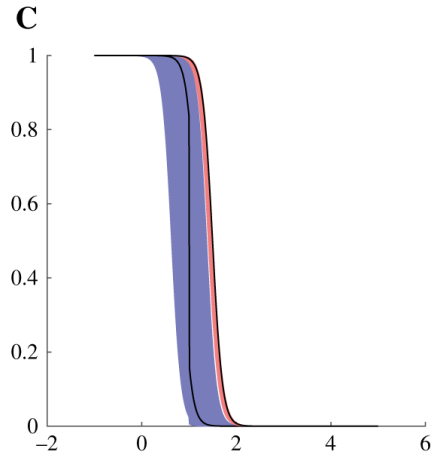

Figure 4: (A) and (B) Prior and posterior distributions of $\gamma^{r}$ and $\gamma$, respectively. (C) Prior (left blue region ) and posterior (right red region) 95\% highest density interval for the transition function conditional on $\gamma^{r}$ and $\gamma$; the black line corresponds to the median transition function.

\subsection{Model inference}

To estimate the model, we iterate 250,000 times over the sampling steps (i)-(iv) listed in Section 3.1, retain every third after a burn-in of 50,000 draws, obtaining $M=66,666$ draws for posterior inference. Figure 5 plots the interest rate and the inflation rate along with the mean posterior probabilities of state $1\left(I_{t}=1\right)$ in yellow. ${ }^{9}$ The estimate discriminates clearly between the two states. State 1 also prevailed at the end of the 1970s, a period where the Swiss franc was also subject to appreciation against the German mark and where therefore, interest rates were also decreased to a then all-time low. The horizontal line indicates the threshold level at $1.5 \%$ inferred from the parameter estimates of the transition function. 


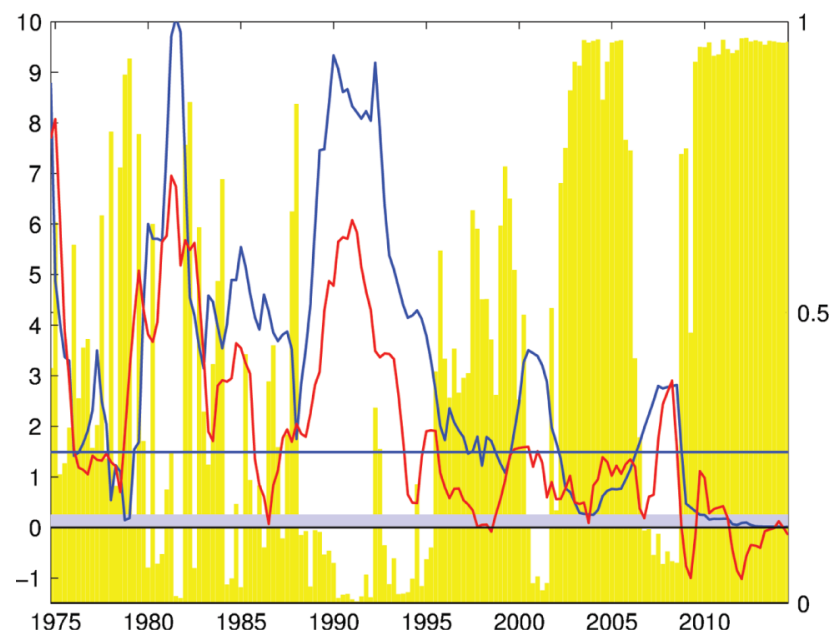

Figure 5: Annual inflation rate (red) and interest rate (blue).

Mean posterior probability (yellow) of state 1 . The periods during which the interest rate is defined to be constrained are those in which the interest rate lies in the shaded area $(b \leq 0.25)$. The horizontal line indicates the inferred threshold level $1.5 \%$ at which $P\left(I_{t}=1\right)=0.5$.

The shaded area below $b=0.25$ indicates in which periods the interest rate is thought to be constrained. On the left-hand side in Figure 6, the observed interest rate is plotted along with the posterior estimates of the latent observations. The areas decreasing in shades correspond to, respectively, the $25 \%$, the $50 \%$ and the $80 \%$ interval of highest posterior density. Compared with the end of the 1970s, the ZLB on the interest rate appears to bind more strongly at the end of the sample given that the median of the latent interest rate decreases to nearly $-0.6 \%$. The figures below the right-hand histograms in Figure 6 show, additionally, that over $90 \%$ of the sampled period-specific latent interest rates, $y_{1 t}^{*}<b$, were lower than the all-time minimum observed value for the interest rate, $\min _{t}\left\{y_{1 t}\right\}$ through $2014 .^{10}$
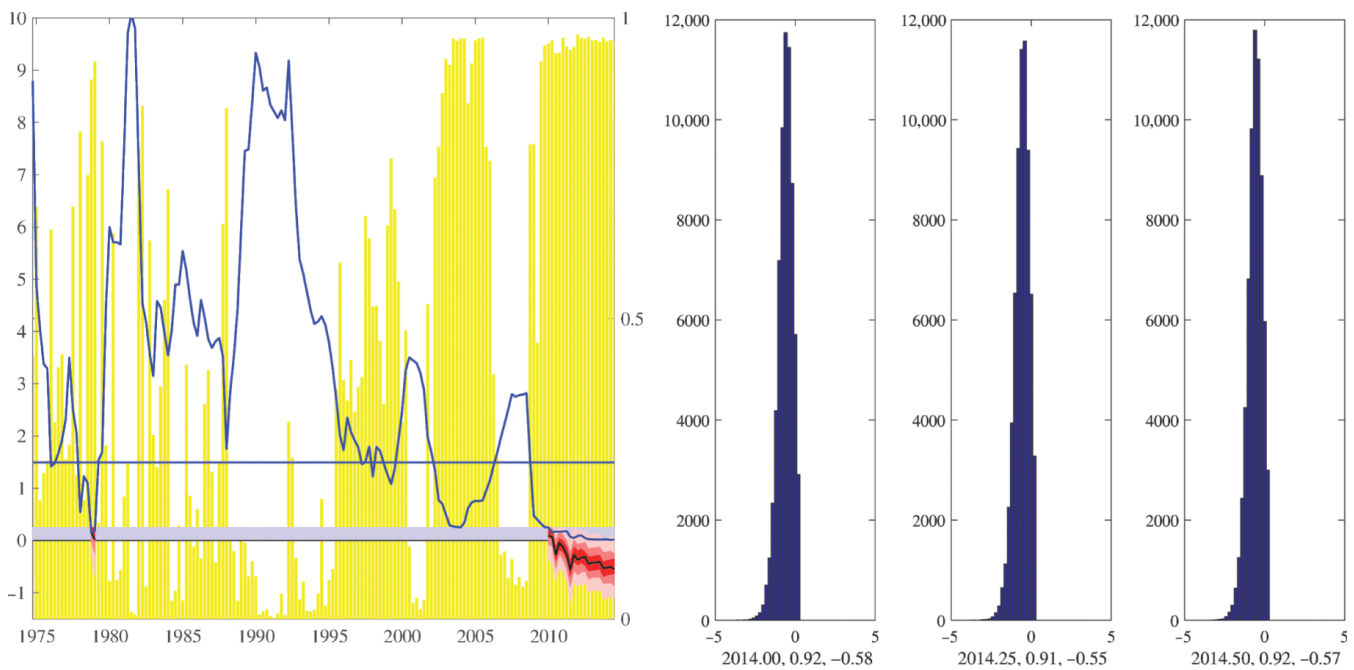

Figure 6: Left-hand: Observed interest rate and model-based estimate of $\boldsymbol{y}_{\mathbf{1}}^{*}<b$ (red); the black line is the median, the areas decreasing in shades correspond to, respectively, the $25 \%$, the $50 \%$ and the $80 \%$ interval of highest posterior density; mean posterior probability of state 1 (yellow). Right-hand: histogram of $y_{1 t}^{*}<b$ for $t=2014.00,2014.25,2014.50$, i.e. 2014 first through third quarter; the second and third numbers under each histogram refer to, respectively, $P\left(y_{1 t}^{*}<\min _{t}\left\{y_{1 t}\right\}\right)$ and median $\left(y_{1 t}^{*}\right)$.

To document that dynamics change when the interest rate enters the critical ZLB region, we plot impulse responses to a structural shock identified as a risk-premium shock. Monetary policy can counter-act the effects of a risk-premium shock, which effects an appreciation for a small open economy, by lowering the interest rate. Obviously, this reaction will be constrained if the interest rate is already very low. As a consequence, the shortterm and the long-term pass-through effects on prices will also differ in the two situations (see also Bäurle and Kaufmann, 2018). To obtain structural identification, we impose sign restrictions on the impact and next period responses of the variables as shown in Table 1 (Arias, Rubio-Ramírez and Waggoner, 2018). A riskpremium shock is expected to appreciate the currency. In a small open economy, the pass-through should lead to a decrease in prices. Monetary policy can counteract the effects by lowering the interest rate. The response 
of GDP is not restricted and all responses are left unrestricted after the first two periods thereby permitting inference on whether the medium-term and the long-term effects differ between the two states.

Table 1: Sign restrictions on the impact and next period responses of the variables.

\begin{tabular}{lllll}
\hline & Reaction in & & \\
\cline { 2 - 5 } Shock to & GDP & CPI & Short rate & exchange rate \\
\hline Risk-premium & - & $\downarrow$ & $\downarrow$ & $\uparrow$ \\
\hline
\end{tabular}

A
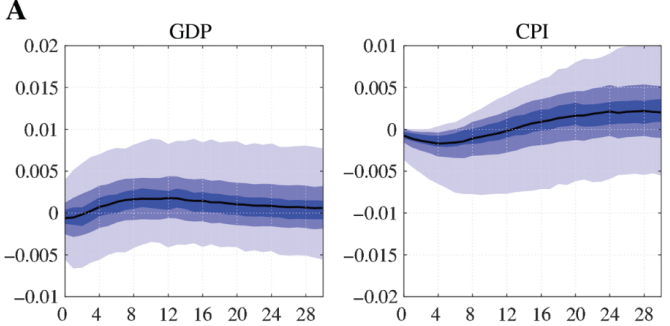

Short rate

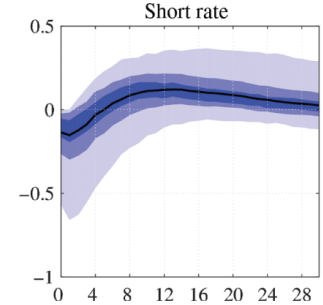

B

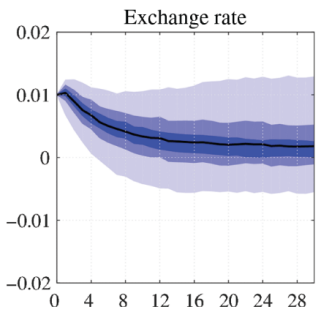

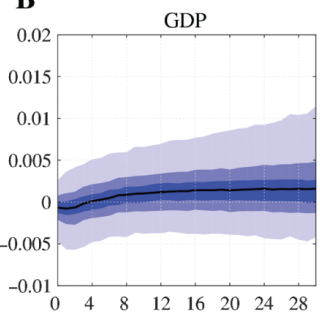

Short rate

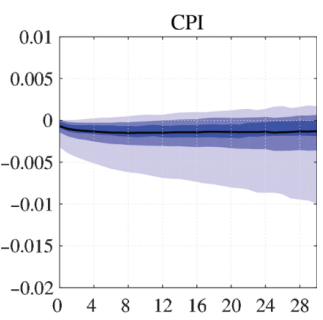

Exchange rate
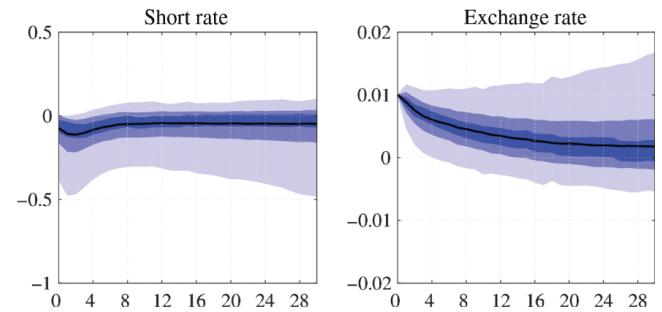

Figure 7: Impulse responses to a risk premium shock identified by sign restrictions and normalized to a corresponding $1 \%$ appreciation shock.

The black line is the median response, the areas decreasing in shades correspond to, respectively, the $25 \%$, the $50 \%$ and the $80 \%$ interval of highest posterior density. (A) $I_{t}=0$. (B) $I_{t}=1$.

The state-specific impulse responses to a risk-premium shock are plotted in Figure 7. The responses are normalized to a $1 \%$ appreciation in the exchange rate. Although the density intervals are quite large, clear patterns are discernible. In the short-term, we observe that there is obviously more leeway for the interest rate to decrease transitorily in state $I_{t}=0$. The response of GDP is not restricted and is generally near zero. Nevertheless, the median shows a positive transitory effect, likely initiated by the decrease in the interest rate. The negative pass-through to prices is transitory and after two years, level-reversion takes place. The long-run effects on prices, however, are very different between the two states. Given that in the ZLB region the interest rate cannot react as strongly to the risk-premium shock, the initial negative pass-through to prices remains permanent. Or in other words, a transitory risk-premium shock translates into a permanent effect on the price level. Although long-run cross-country relationships are not modelled explicitly, we know that for a given real exchange rate, permanent negative effects on the price level induce further long-run nominal appreciation on the currency.

Figure 8 and Table 2 evaluate the differences between state-specific impulse responses more explicitly. We compute the differences using 7341 (11\% of all) draws accepted across both states. In state $I_{t}=1$, the median negative price response is lower after about two years and remains persistently low over the long-run. The difference widens to $0.5 \%$ after 28 quarters. After half a year, the interest rate response is lower by a median of 13 basis points (bps) in state $I_{t}=1$. The difference widens to $19 \mathrm{bps}$, reflecting the inability to counteract the appreciation shock at the ZLB. A slight reversion to a difference of $14 \mathrm{bps}$ is observable in the long run only. The lower interest rate and CPI responses in state $I_{t}=1$ explain why GDP responses do not differ across both states. Likewise, the persistence of the risk premium shock (the exchange rate responses) shock is virtually the same in both states. ${ }^{11}$

Overall, these results are consistent with those presented in Bäurle and Kaufmann (2018), although we obtain a weaker negative CPI response. ${ }^{12}$ However, empirical evidence for other countries also suggests that the ZLB leads to a gradual but persistent effect on macroeconomic variables (see e.g. Caggiano, Castelnuovo and Pellegrino, 2017). 

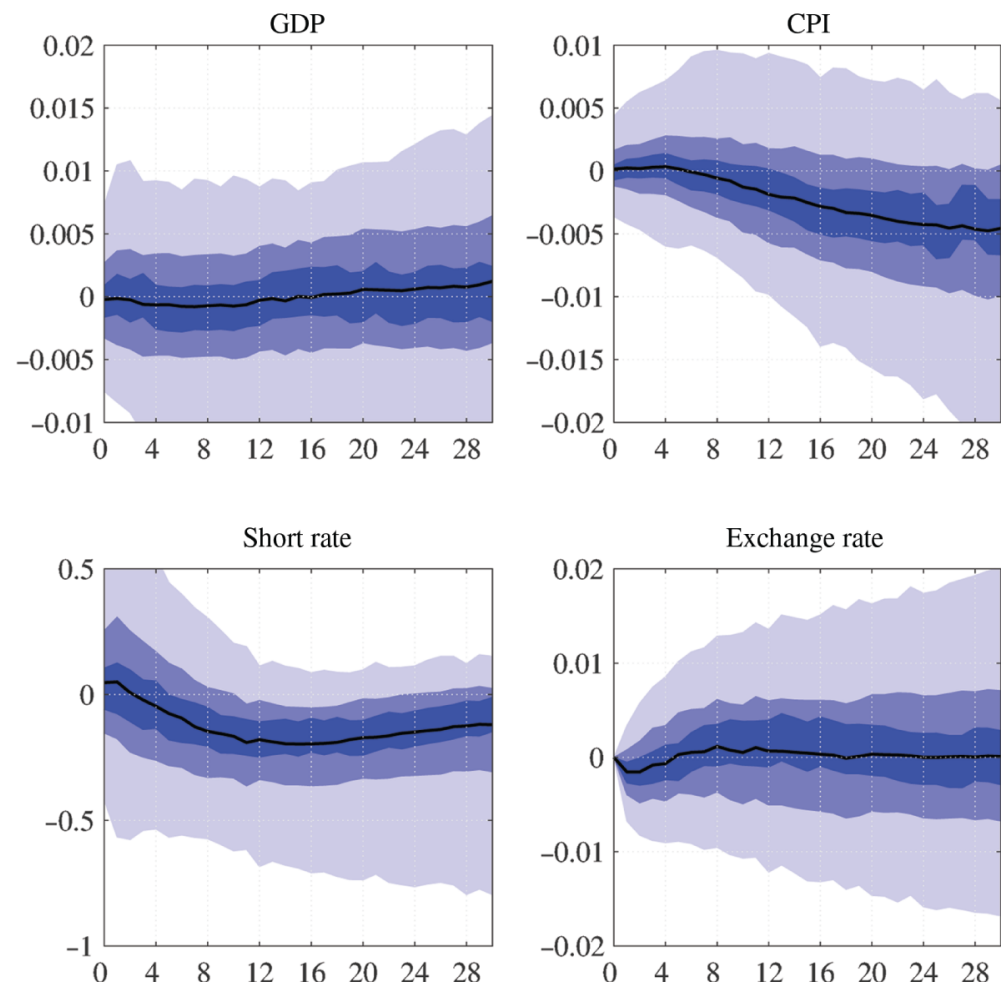

Figure 8: Difference in impulse responses: State $I_{t}=1-$ State $I_{t}=0$ response; computed with 7341 (11\% of all) draws accepted simultaneously across both states.

Response to a risk premium shock identified by sign restrictions and normalized to a corresponding $1 \%$ appreciation shock. The black line is the median response, the areas decreasing in shades correspond to, respectively, the $25 \%$, the $50 \%$ and the $80 \%$ interval of highest posterior density.

Table 2: Difference in impulse responses: State $I_{t}=1-$ State $I_{t}=0$ response; computed with 7341 (11\% of all) draws accepted simultaneously across both states.

\begin{tabular}{|c|c|c|c|c|}
\hline Horizon & GDP & CPI & Short rate & Exchange rate \\
\hline \multirow[t]{2}{*}{1} & -0.0002 & 0.0001 & 0.0579 & 0 \\
\hline & $(-0.0030,0.0027)$ & $(-0.0014,0.0015)$ & $(-0.1250,0.2668)$ & - \\
\hline \multirow[t]{2}{*}{4} & -0.0006 & 0.0003 & -0.0275 & -0.0011 \\
\hline & $(-0.0047,0.0031)$ & $(-0.0017,0.0025)$ & $(-0.2425,0.1919)$ & $(-0.0046,0.0025)$ \\
\hline \multirow[t]{2}{*}{8} & -0.0007 & -0.0004 & -0.1283 & 0.0004 \\
\hline & $(-0.0046,0.0033)$ & $(-0.0032,0.0027)$ & $(-0.3128,0.0445)$ & $(-0.0043,0.0049)$ \\
\hline \multirow[t]{2}{*}{12} & -0.0007 & -0.0014 & -0.1816 & 0.0007 \\
\hline & $(-0.0047,0.0032)$ & $(-0.0047,0.0023)$ & $(-0.3479,-0.0344)$ & $(-0.0046,0.0059)$ \\
\hline \multirow[t]{2}{*}{16} & -0.0001 & -0.0026 & -0.1924 & 0.0006 \\
\hline & $(-0.0042,0.0041)$ & $(-0.0065,0.0014)$ & $(-0.3553,-0.0517)$ & $(-0.0051,0.0063)$ \\
\hline \multirow[t]{2}{*}{20} & 0.0004 & -0.0033 & -0.1820 & 0.0002 \\
\hline & $(-0.0038,0.0049)$ & $(-0.0076,0.0010)$ & $(-0.3491,-0.0395)$ & $(-0.0060,0.0063)$ \\
\hline \multirow[t]{2}{*}{24} & 0.0011 & -0.0044 & -0.1678 & 0.0006 \\
\hline & $(-0.0032,0.0060)$ & $(-0.0092,0.0000)$ & $(-0.3440,-0.0257)$ & $(-0.0055,0.0075)$ \\
\hline \multirow[t]{2}{*}{28} & 0.0016 & -0.0050 & -0.1398 & 0.0003 \\
\hline & $(-0.0029,0.0068)$ & $(-0.0103,-0.0005)$ & $(-0.3214,0.0092)$ & $(-0.0064,0.0072)$ \\
\hline
\end{tabular}

Response to a risk premium shock identified by sign restrictions and normalized to a corresponding $1 \%$ appreciation shock. The median difference ( $50 \%$ highest posterior density) in log levels, except for the short rate (percentage points).

\subsection{Scenario-based forecasts}

By implementing Scenario 2. (iii), the model estimate is used to answer the following questions. Where does the system drift to if the mean interest rate is observed to fall to $-1 \%$ in the first quarter of the forecast horizon and remain at this level for one year? What is the probability of exiting the ZLB critical region and under what 
economic conditions does this happen? These questions may be relevant against the background of the SNB's decision to introduce negative interest rates at the end of 2014. However, it is important to recognize that our scenario does not implement a policy experiment, i.e. it does not provide an estimate of the causal impact of a decrease in interest rates to $-1 \%$. It merely describes the economic conditions consistent with an average interest rate at $-1 \%$ for one year. ${ }^{13}$

The forecast horizon is 6 years, $H=24$. The sample from the forecasting density (9) is obtained by producing dynamic forecasts using all posterior parameter draws. The left-hand side in Figure 9 displays the forecasts we obtain if the mean interest rate stays at a level of $-1 \%$ for one year from 2014Q4 onwards and is held stable at lagged forecasts afterwards. Over the whole forecasting period, the mean interest rate remains stable in this scenario. However, there is a relevant chance (12\%) for the system to exit the ZLB region. Year-on-year GDP growth and inflation are low on average, but still positive $(1.2 \%$ and $0.1 \%$, respectively). This overall stable development is accompanied by further appreciation pressures.

The right-hand side in Figure 9 plots the forecasts of those 12\% paths that finally exit the ZLB region again. On average, year-on-year GDP growth and inflation reach, respectively, $1.4 \%$ and $0.9 \%$, and we observe that they are accelerating over the forecast period. At the end of the forecast horizon year-on-year GDP growth reaches $3 \%$ and inflation $3.8 \%$. At the same time, the appreciation trend is broken. On average, appreciation still amounts to $0.2 \%$, while towards the end of the forecast horizon depreciation increases to $3.3 \%$. Thus, the results indicate that economic conditions have to improve quite substantially to permanently exit the ZLB regime. In contrast to the previous results, an exit from the ZLB region would be accompanied by a depreciation of the Swiss franc.
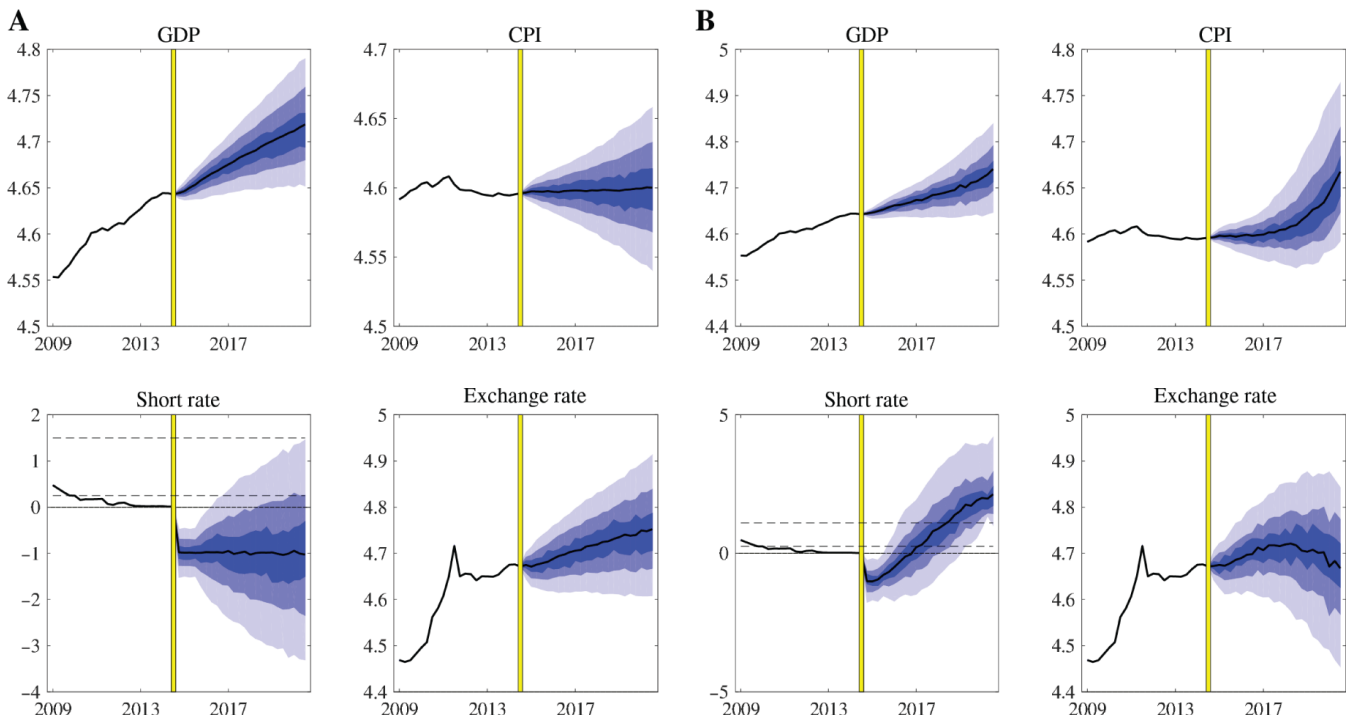

Figure 9: Conditional forecast distributions.

The black line is the median forecast, the areas decreasing in shades correspond to, respectively, the $25 \%$, the $50 \%$ and the $80 \%$ interval of highest forecast density. The vertical line denotes the end of the sample, 2014Q3. (A) Forecast distribution conditional on a mean interest rate lowered to $-1 \%$ for 1 year. (B): Conditional forecast distribution for those paths that exit the ZLB region (mean $12 \%$ probability), $\boldsymbol{I}_{T+H}=\mathbf{0}$

On exiting the ZLB region, our model is slightly more optimistic than professional forecasters' expectations about a positive libor. Figure 10 shows the share of survey participants in the KOF Consensus Survey that expect a positive three-month libor one and twelve months ahead. From 2015 onwards, this share hovers slightly below $10 \%$ which indicates that participants expected the SNB to exit the negative interest rate regime, if at all, a bit later than what the model predicts (12\%). 


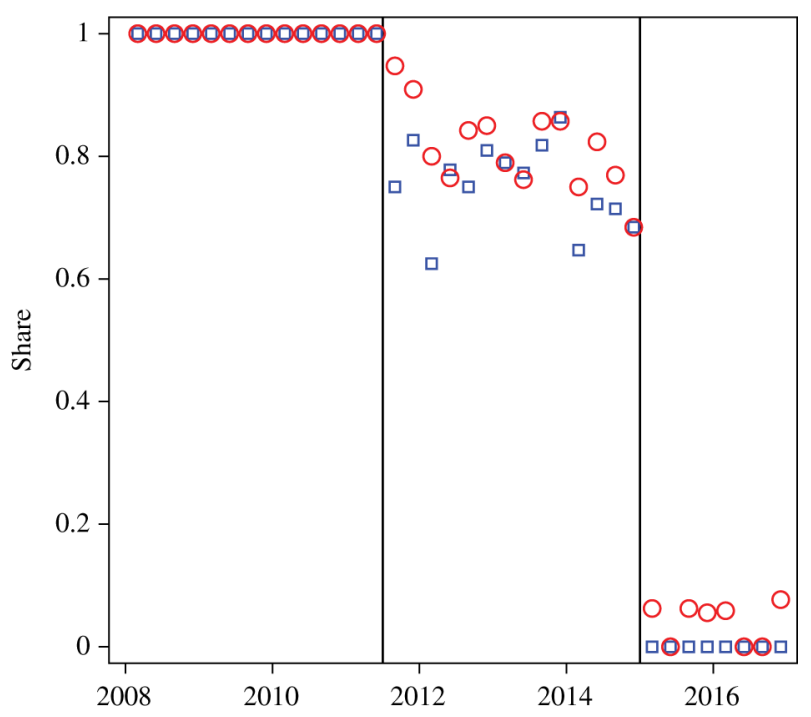

Figure 10: KOF Consensus Survey.

Share of professional forecasters expecting a positive libor 3-months (blue) and 12-months (red) ahead. The vertical lines indicate the introduction and removal of a minimum exchange rate against the euro.

\section{Stochastic instead of regime switching volatility}

\subsection{Alternative models}

Work by Clark (2011) and Diebold, Schorfheide, and Shin (2017), among others, show that macroeconomic data display a large amount of changing volatility. Our model is restrictive insofar as volatility is fixed within each regime. In order to assess the implication of the regime switching specification, we compare our model (the Full model henceforth) to one which generalizes to stochastic volatility (SV henceforth), re-specifying $\Sigma_{t}$ in (2) as:

$$
\Sigma_{t}=A_{t}^{-1} H_{t} A_{t}^{-1^{\prime}}
$$

with $H_{t}$ diagonal, $H_{t}=\operatorname{diag}\left(\exp \left(\sigma_{1 t}\right), \ldots, \exp \left(\sigma_{N t}\right)\right)$ and $A_{t}$ lower diagonal with ones on the diagonal. As usual, we specify independent random walk processes for the $\log$ volatilities $\sigma_{t}=\left(\sigma_{1 t}, \ldots, \sigma_{N t}\right)^{\prime}$ and the structural correlations $\mathbf{a}_{t}=\left\{a_{i j, t} \mid i=2, \ldots, N ; j=1, \ldots, i-1\right\}$ :

$$
\begin{aligned}
& \sigma_{t}=\sigma_{t-1}+\zeta_{t}, \zeta_{t} \sim N\left(0, \Omega_{\sigma}\right) \\
& \mathbf{a}_{t}=\mathbf{a}_{t-1}+\eta_{t}, \eta_{t} \sim N\left(0, \Omega_{\mathrm{a}}\right)
\end{aligned}
$$

Following the setup of Chan and Eisenstat (2018), we treat initial conditions $\sigma_{0}$ and $\mathbf{a}_{0}$ as parameters to estimate, assuming normal prior distributions $N\left(0,10 I_{k}\right)$, with $I_{k}$ being the identity matrix of dimension $k=\{N, N(N-$ 1) /2\} for, respectively, $\sigma_{0}$ and $\mathbf{a}_{0}$. Elements of innovation variances $\Omega_{\sigma}$ and $\Omega_{\mathrm{a}}$ are independently inverse Gamma distributed, $\omega_{\sigma j} \sim \operatorname{IG}\left(5, S_{\omega_{\sigma}}\right), j=1, \ldots, N$, and $\omega_{\mathrm{a} j} \sim \operatorname{IG}\left(5, S_{\omega_{\mathrm{a}}}\right), j=1, \ldots N(N-1) / 2$, respectively. The scales $S_{\omega_{\sigma}}$ and $S_{\omega_{\mathrm{a}}}$ are specified such to obtain a prior mean of $0.1^{2}$ and $0.01^{2}$ for, respectively, $\omega_{\sigma j}$ and $\omega_{\mathrm{a} j}$.

In empirical work we often find strong evidence that intercepts are significantly time-varying while evidence for time-varying autoregressive coefficients is much weaker (Belmonte, Koop and Korobilis, 2014). Against this background, we additionally compare the Full model with a model including stochastic volatility, switching intercept and constant autoregressive coefficients (Intercept and SV henceforth). The compact form of restricted model (1) is written as

$$
y_{t}=\mu_{t}+X_{t} \beta+\varepsilon_{t}, \varepsilon_{t} \sim N\left(0, \Sigma_{t}\right)
$$


with time-invariant $\beta=\operatorname{vec}\left(\left[B_{1}, \ldots, B_{p}\right]^{\prime}\right), \Sigma_{t}=A_{t}^{-1} H_{t} A_{t}^{-1^{\prime}}$ specified as above and intercepts $\mu_{t}=\mu_{0}\left(1-I_{t}\right)+$ $\mu_{1} I_{t}$ the only parameters subject to regime switches.

Note that both models are estimated with the Tobit specification as defined in (1). A Tobit specification combined with stochastic volatility and regime switching in (some) parameters is, up to our knowledge, unique in applied econometric studies.

Define $\hat{y}_{t}=y_{t}^{*}-X_{t} \beta_{t}$, i.e. condition on right-hand side variables in (1), and represent the conditional model in structural form:

$$
\begin{aligned}
A_{t} \hat{y}_{t} & =v_{t}, v_{t} \sim N\left(0, H_{t}\right) \\
\hat{y}_{t} & =\hat{\mathbf{W}}_{t} \mathbf{a}_{t}+v_{t}
\end{aligned}
$$

with

$$
\hat{\mathbf{W}}_{t}=\left[\begin{array}{cccccccc}
0 & \ldots & & & & & & 0 \\
-\hat{y}_{1 t} & 0 & & \ldots & & & & 0 \\
0 & -\hat{y}_{1 t} & & -\hat{y}_{2 t} & 0 & \ldots & & 0 \\
\vdots & & 0 & & \ddots & & & \vdots \\
0 & \ldots & & & & -\hat{y}_{1 t} & \ldots & -\hat{y}_{N-1, t}
\end{array}\right]
$$

Based on (15), we adjust the sampler in Section 3.1 to estimate the model generalized to stochastic volatility in (11) and (14). For both setups, model parameter $\theta$ is expanded to include $\Sigma_{t}$ and hyperparameters, $\theta_{\mathrm{SV}}=\left\{\beta_{k}, \Sigma_{t}, \boldsymbol{\gamma}, \boldsymbol{\sigma}_{0}, \mathbf{a}_{0}, \Omega_{\sigma}, \Omega_{\mathrm{a}} \mid k=0,1, \boldsymbol{\gamma}=\left(\boldsymbol{\gamma}^{\mathrm{r}}, \boldsymbol{\gamma}\right)\right\}$ and $\theta_{\mathrm{IwSV}}=\left\{\mu_{k}, \beta, \Sigma_{t}, \boldsymbol{\gamma}, \boldsymbol{\sigma}_{0}, \mathbf{a}_{0}, \Omega_{\sigma}, \Omega_{\mathrm{a}} \mid k=0,1, \boldsymbol{\gamma}=\left(\gamma^{\mathrm{r}}, \boldsymbol{\gamma}\right)\right\}$, respectively. When generalized to stochastic volatility, sampling step (iv) becomes: ${ }^{14}$

(iv.SV) (b) $\pi\left(\boldsymbol{\sigma} \mid \hat{\mathbf{y}}, \mathbf{a}, \Omega_{\sigma}, \sigma_{0}\right)$ and $\pi\left(\sigma_{0} \mid \hat{\mathbf{y}}, \mathbf{a}, \sigma_{1}, \Omega_{\sigma}\right)$

(c) $\pi\left(\mathbf{a} \mid \hat{y}, \boldsymbol{\sigma}, \Omega_{\mathrm{a}}, \mathbf{a}_{0}\right)$ and $\pi\left(\mathbf{a}_{0} \mid \hat{y}, \boldsymbol{\sigma}, \mathbf{a}_{1}, \Omega_{\mathrm{a}}\right)$

(d) $\pi\left(\Omega_{\sigma} \mid \hat{\mathbf{y}}, \boldsymbol{\sigma}, \sigma_{0}\right)$ and $\pi\left(\Omega_{\mathrm{a}} \mid \hat{\mathbf{y}}, \mathbf{a}, \mathrm{a}_{0}\right)$

where again, bold-faced values gather all observations of a vector or matrix, e.g. $\Sigma=\left\{\Sigma_{1}, \ldots \Sigma_{T}\right\}$ or $\mathbf{a}=$ $\left\{\mathrm{a}_{1}, \ldots, \mathrm{a}_{T}\right\}$.

For estimating model (14), step (a) in (iv.SV) is adjusted to

(iv.ISV) (a) $\pi\left(\mu_{0}, \mu_{1}, \beta \mid \mathbf{y}^{*}, \mathbf{X}, \mathbf{I}, \boldsymbol{\Sigma}\right)$

while steps (b)-(d) remain the same with re-defined $\hat{y}_{t}=y_{t}^{*}-\mu_{t}-X_{t} \beta$.

Sampler convergence for the SV and Intercept with SV models is much slower. Therefore, we iterate 700,000 times over the sampler, retaining every third of the last 200,000 draws, i.e. 66,666 draws for posterior inference.

\subsection{Model comparison}

We evaluate the log predictive score and the Bayesian information criterion (BIC) jointly for GDP and CPI to compare the model fit of the Full model with the SV and Intercept and SV models. We choose these two variables because they represent Swiss monetary policy's main focus and we observe all values over the sample period.

Table 3 documents that the Full model performs significantly better in terms of log predictive score and BIC than the alternative specifications. This applies even though we favour both models with SV when determining the penalty term. We count each of the $N(N+1) / 2$ elements of $\Sigma_{t}$ as one parameter neglecting its time variation and also neglect counting the estimated elements of $\Omega_{\sigma}$ and $\Omega_{\mathrm{a}}$. Intercept and SV performs slightly better than SV. Figure 11 plots time-specific values of the log-predictive scores (Panel (a)) and the log determinant $\log \left|\sum_{t, \mathrm{GPD}, \mathrm{CPI}}\right|($ Panel (b)). Obviously, the Full model achieves a higher log predictive score in most periods. The log predictive score is considerably worse only in quarters where large shocks occurred: In 1974 and 2008 when, respectively, the oil price and the financial crisis shock hit the economy. This evidence in favour of the Full model is reflected in Figure 12. Overall, volatility evolves smoothly over time. In particular for GDP and CPI, volatilities remain fairly constant over time. For the three-month libor and the exchange rate, volatilities also remain quite stable after adjusting to lower levels over time.

Table 3: Average log predictive score and BIC; evaluated for GDP and CPI.

\begin{tabular}{lllllll}
\hline Model & \multicolumn{2}{c}{ Log predictive score } & & & BIC \\
\cline { 2 - 3 } & Mean & Median & & $\sum_{t} \log \left|\sum_{t, \mathrm{GDP}, \mathrm{CPI}}\right|$ & Penalty & BIC \\
\hline
\end{tabular}




\begin{tabular}{|c|c|c|c|c|c|}
\hline Full & 1.26 & 1.26 & -3473.7 & $42 \log (160)$ & -3260.5 \\
\hline SV & 1.11 & 1.11 & -2879.4 & $39 \log (160)$ & -2681.5 \\
\hline $\begin{array}{l}\text { Intercept and } \\
\text { SV }\end{array}$ & 1.11 & 1.11 & -2890.2 & $23 \log (160)$ & -2773.5 \\
\hline
\end{tabular}

Model with switching parameters and error covariance matrix (Full), switching parameters and stochastic volatility (SV) and switching intercept, constant AR parameters and stochastic volatility (Intercept and SV).
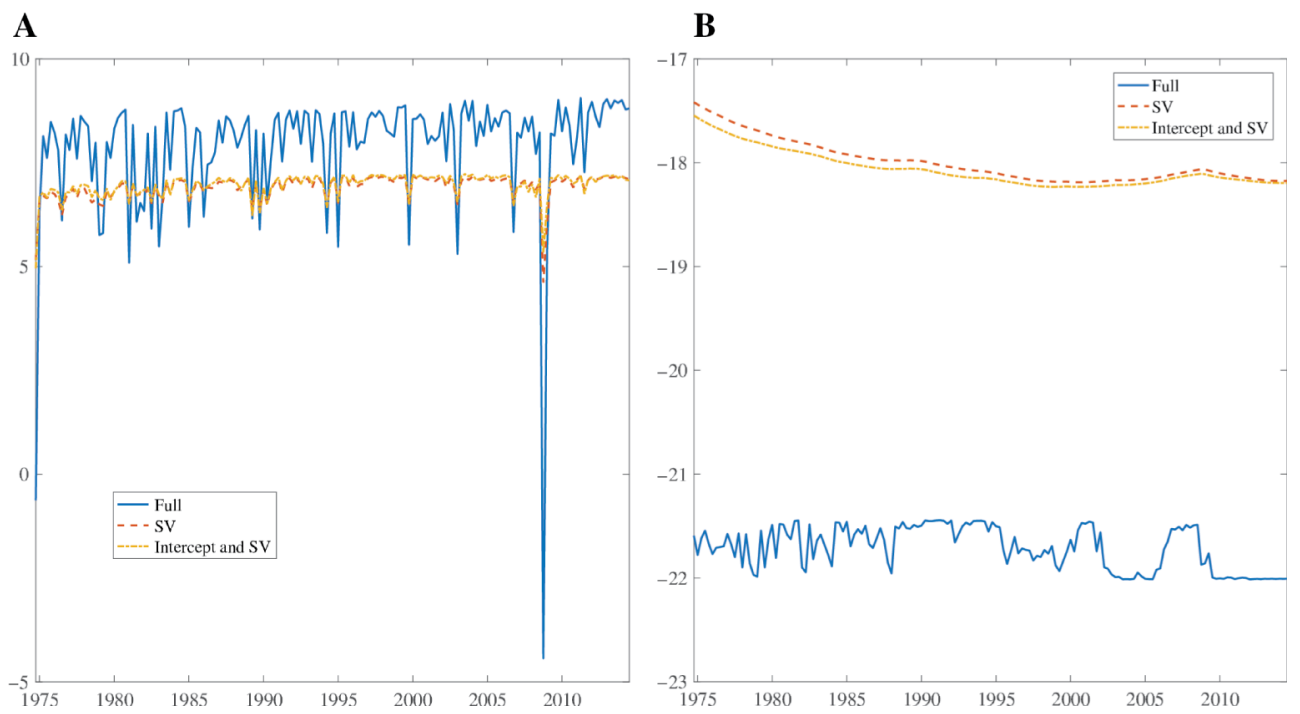

Figure 11: Regime switching against stochastic volatility.

Evaluations for GDP and CPI. Model with switching parameters and error covariance matrix (Full), switching parameters and stochastic volatility (SV) and switching intercept, constant AR parameters and stochastic volatility (intercept and SV). (A) Mean log predictive score. (B) Mean $\log \left|\Sigma_{t, \mathrm{GDP}, \mathrm{CPI}}\right|$.
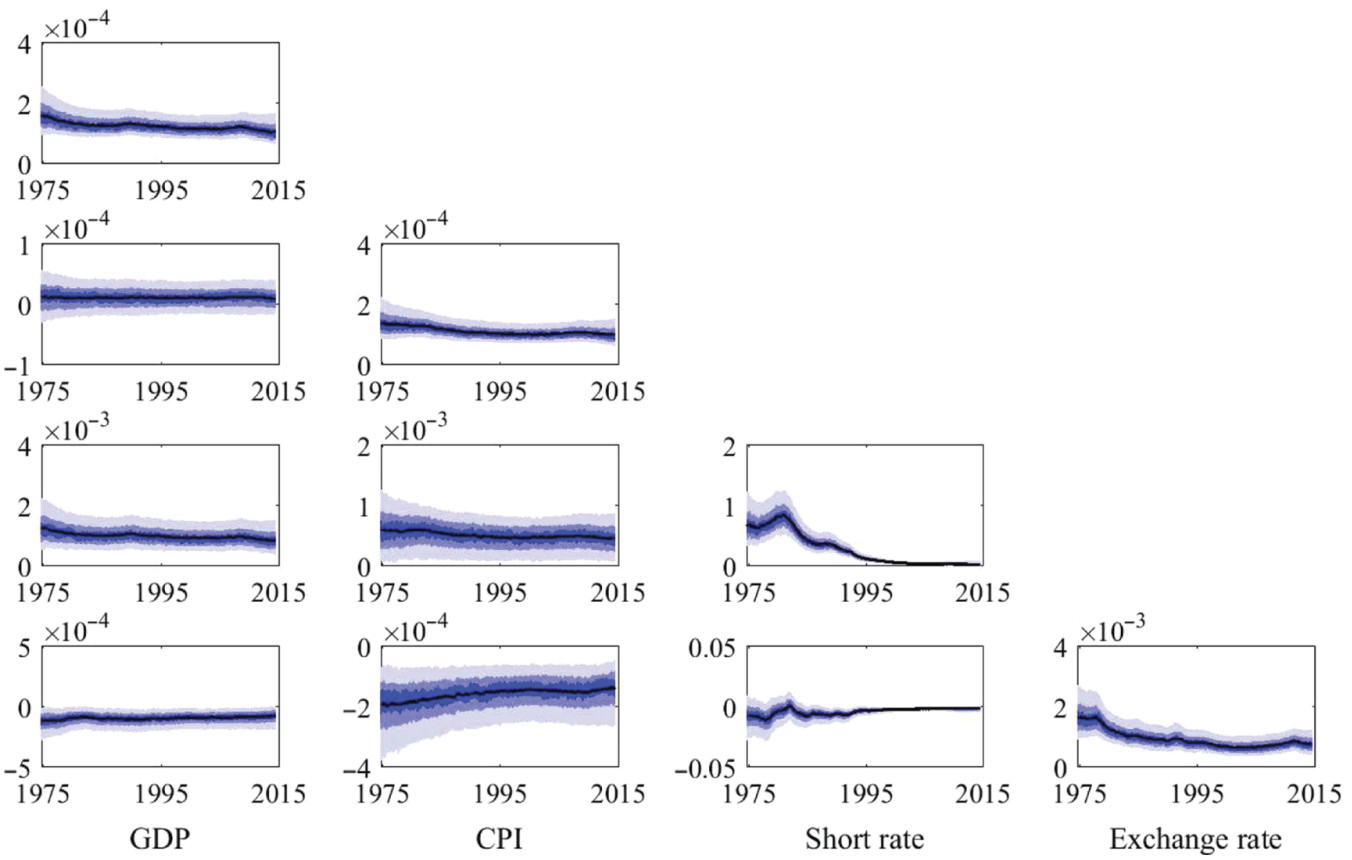

Figure 12: Stochastic volatility estimates.

Lower diagonal of $\Sigma_{t}$. The shaded areas refer to the $25 \%, 50 \%$ and $80 \%$ highest posterior density interval.

\subsection{Selected results for the SV model}

Although achieving a lower model fit, the results obtained from the SV model confirm those of the Full model. Figure 13 and Figure 14 show the analogue of Figure 7 and Figure 8, respectively. ${ }^{15}$ Generalizing to stochastic volatility, increases estimation uncertainty, in particular in state $I_{t}=1$. The main conclusions remain unchanged, 
however. Near the ZLB, a risk-premium shock has a persistent negative effect on CPI, and the short rate remains persistently lower as well. As in the Full model, GDP's responses do not differ significantly between regimes, while the shock reflects less persistently in the exchange rate. ${ }^{16}$

A
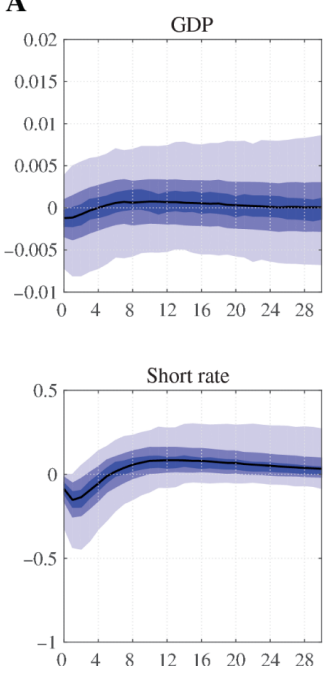
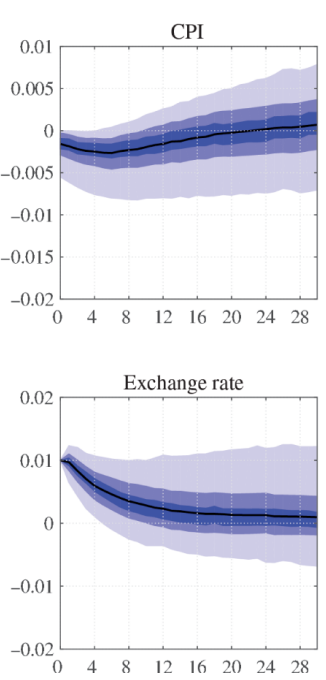

B
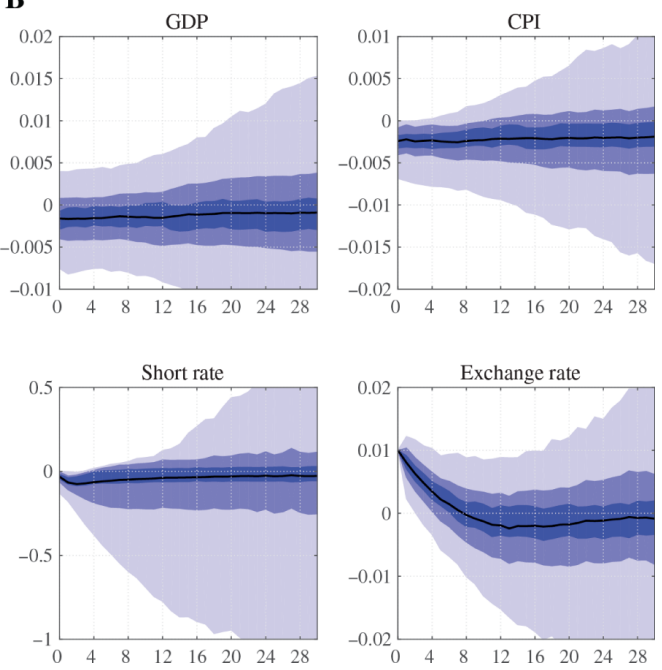

Figure 13: Switching VAR with SV.

Impulse responses to a risk premium shock identified by sign restrictions and normalized to a corresponding $1 \%$ appreciation shock. The black line is the median response, the areas decreasing in shades correspond to, respectively, the $25 \%$, the $50 \%$ and the $80 \%$ interval of highest posterior density. (A) $I_{t}=0, \Sigma_{t, 199101}$. (B) $I_{t}=1, \Sigma_{t, 200502}$.
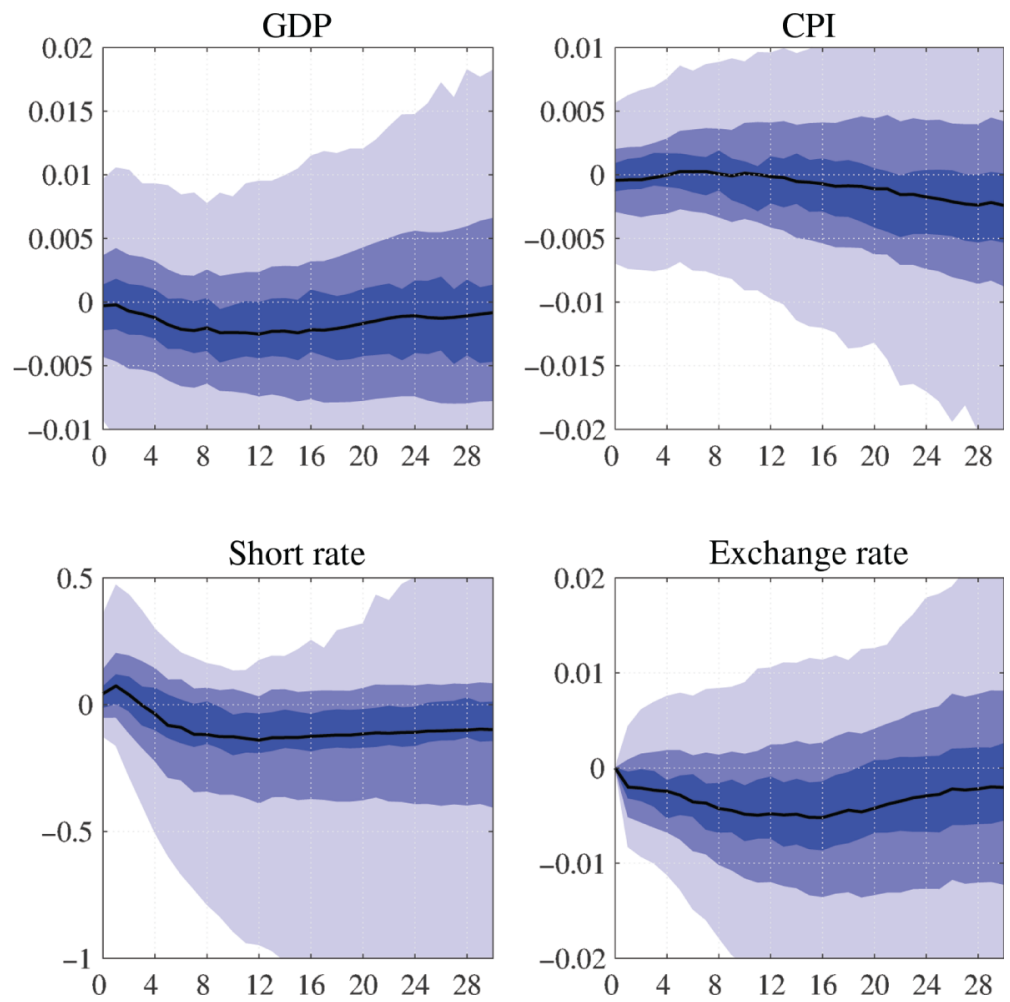

Figure 14: Switching VAR with SV.

Difference in impulse responses: State $I_{t}=1-$ State $I_{t}=0$ response; $8801(13 \%)$ accepted draws across both states. Response to a risk premium shock identified by sign restrictions and normalized to a corresponding $1 \%$ appreciation shock. The black line is the median response, the areas decreasing in shades correspond to, respectively, the $25 \%$, the $50 \%$ and the $80 \%$ interval of highest posterior density.

Figure 15 shows the analogue to Figure 6. The posterior mean probabilities of $I_{t}=1$ do not differ much compared with the Full model. Again stochastic volatility introduces more estimation uncertainty, which is reflected in a more dispersed posterior distribution for $y^{*}$. Overall, the SV model estimates a decrease in the median latent short rate to -0.74 to the end of the sample, compared with -0.57 for the Full model. Qualitatively, the conditional forecasts (Figure 16) are relatively similar to the Full model. Quantitatively, compared 
with the Full model, average year-on-year GDP growth and inflation are forecasted to be somewhat lower ( $1.0 \%$ compared with $1.2 \%$ ) and higher (0.3\% compared with $0.1 \%)$, respectively. As in the Full model, this development is accompanied by further appreciation pressures (left-hand in Figure 16). The SV model predicts a lower probability of exiting the ZLB region, $2 \%$ compared with $12 \%$ in the Full model. Those draws exiting the ZLB region (right-hand in Figure 16) show a more subdued development than in the Full model. On average, year-on-year GDP growth is $0.6 \%$ and CPI inflation $0.3 \%$ compared with $1.4 \%$ and $0.9 \%$ in the Full model, respectively.
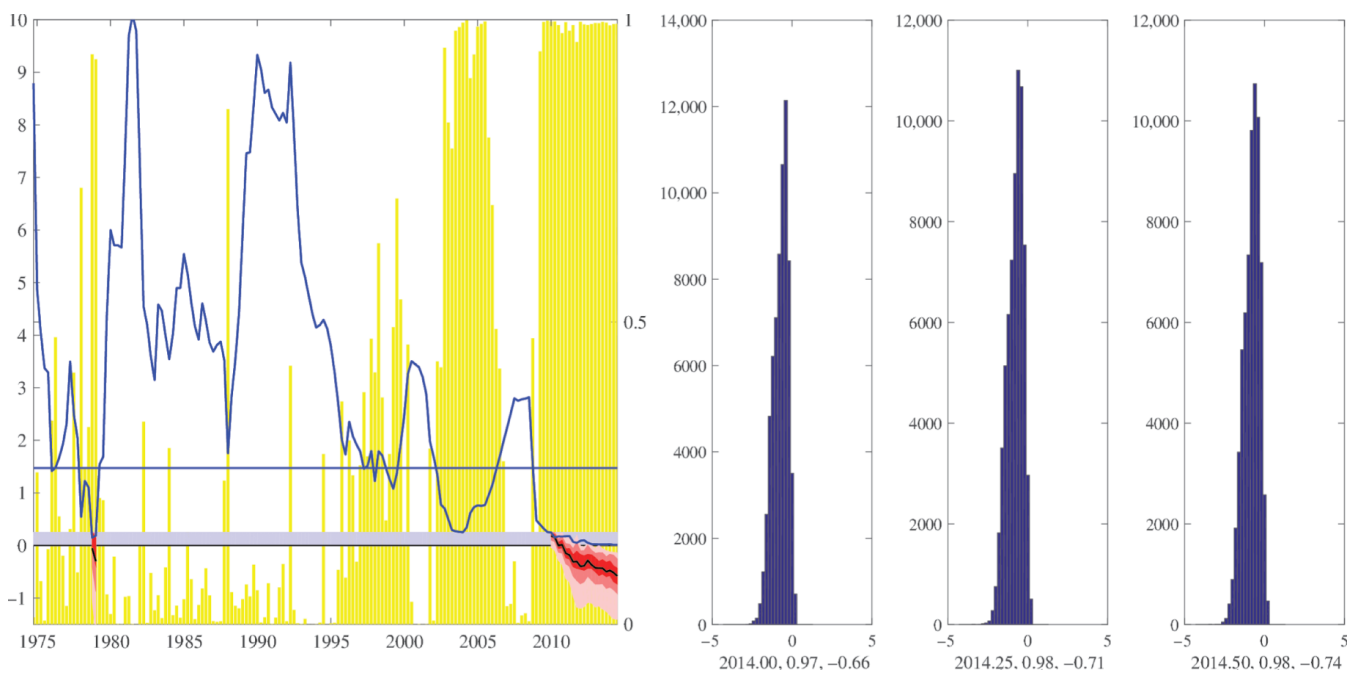

Figure 15: Switching VAR with SV.

Left-hand: Observed interest rate and model-based estimate of $y_{1}^{*}<b$ (red); mean posterior probability of state 1 (yellow). Right-hand: histogram of $y_{1 t}^{*}<b$ for $t=2014.00,2014.25,2014.50$, i.e. 2014 first through third quarter; the second and third numbers under each histogram refer to, respectively, $P\left(y_{1 t}^{*}<\min _{t}\left\{y_{1 t}\right\}\right)$ and median $\left(y_{1 t}^{*}\right)$.
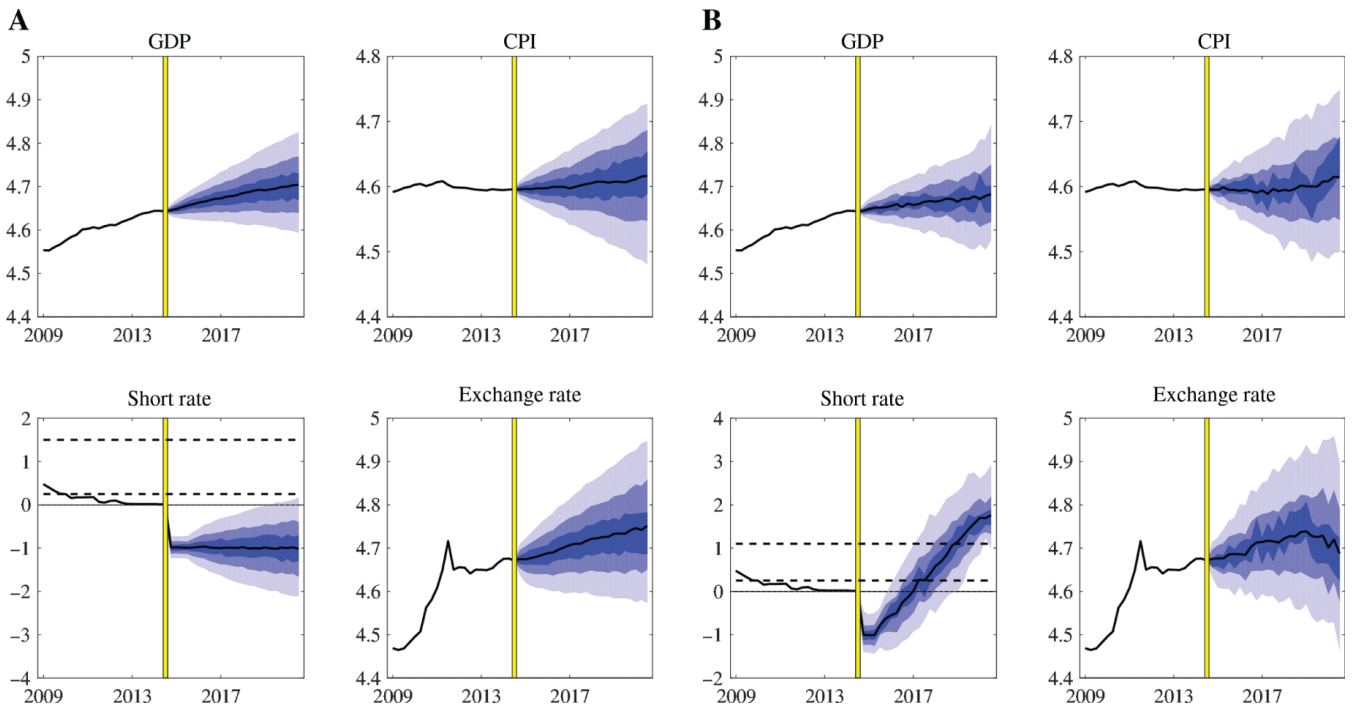

Figure 16: Switching VAR with SV.

The black line is the median forecast, the areas decreasing in shades correspond to, respectively, the $25 \%$, the $50 \%$ and the $80 \%$ interval of highest forecast density. The vertical line denotes the end of the sample, 2014Q3. (A): Forecast distribution conditional on a mean interest rate lowered to $-1 \%$ for 1 year $\left(\Sigma_{T+h}=\Sigma_{T}, h=1, \ldots, 4\right)$. (B): Conditional forecast distribution for those paths that exit the ZLB region (mean $2 \%$ probability).

\section{Conclusion}

In the present paper, we propose to capture changing dynamics between variables near the ZLB with the use of a nonlinear model. A latent state indicator determines the changes in parameters and error covariances of a VAR model. The probability of state transitions depends on the lagged level of the interest rate, which is a natural candidate to indicate whether the system is away from or in the so-called critical ZLB region. It is 
obvious that other variables determining the policy stance like GDP growth or the inflation rate could also be used as covariates. For a small open economy, an alternative could be to include a monetary condition index, which determines the monetary stance by a weighted average of the interest rate and the exchange rate. The specification of the VAR model takes into account that in the ZLB region, the interest rate might be a constrained variable. The estimation of the model then provides us with an inference on the latent rate, i.e. the lower than observed level of the interest rate which would be state- and model-consistent.

We setup a model for four Swiss variables, namely GDP, CPI, the three-month Swiss franc libor and a tradeweighted effective exchange rate. We estimate it within a Bayesian framework, which allows to handle the situation of few observations near the ZLB. Also, we can input subjective information into the specification of prior distributions. For example, a notion for an upper and a lower bound for the interest rate at which we think that dynamics may change, i.e. a prior notion on the threshold value, can be included into the prior of the parameters of the state probability distribution. The results show that dynamics indeed change when the interest rate enters the ZLB region. The impulse response analysis gives evidence that transitory risk-premium shocks which correspond to a $1 \%$ appreciation in the exchange rate translate into a permanent negative price level effect when the interest rate is in the ZLB region. This differs from the normal situation, in which the negative price level effect is transitory.

The endogenous specification of the state probability distribution allows to dynamically forecast the state and the VAR system into the future. In particular, we can evaluate the probability with which the system exits the ZLB region based on its own dynamics. We find that there is a relevant chance to exit the ZLB after 2014. However, this needs to be accompanied by substantial improvements in the economic environment. The model developed in this paper performs well compared to other reasonable alternatives in terms of both fit (as measured by BIC) and forecasting performance.

The model used in the present paper can be extended in various ways. The model for a small open economy would be completed by including a set of foreign, exogenous variables. Additional scenarios could then be evaluated, like the reaction to a further increase or decrease in the foreign policy rate, or a protracted recovery abroad. Another avenue would be to model explicitly long-run common trending behavior among the variables. An issue that is not addressed in the paper is how to identify a monetary policy shock in the ZLB region. Further research will address these extensions.

\section{Acknowledgement}

We thank for constructive comments of the Editor, Associate Editor and two anonymous referees. Rodney Strachan acknowledges the support from the Australian Research Council, Discovery Project DP180102373.

\section{A Appendix}

\section{Distributional properties of censored and uncensored variables}

Given the normality assumption on $\varepsilon_{t}$, model (1) defines a joint normal distribution for the variables $y_{t}^{*}=$ $\left[y_{1 t}^{* \prime}, y_{2 t}^{\prime}\right]^{\prime}$, where $y_{2 t}$ gathers the uncensored variables.

$$
\left[\begin{array}{l}
y_{1 t}^{*} \\
y_{2 t}
\end{array}\right] \mid X_{t}, I_{t}, \theta \sim N\left(\left[\begin{array}{l}
m_{1 I_{t}} \\
m_{2 I_{t}}
\end{array}\right],\left[\begin{array}{cc}
\Sigma_{11, I_{t}} & \Sigma_{12, I_{t}} \\
\Sigma_{21, I_{t}} & \Sigma_{22, I_{t}}
\end{array}\right]\right)
$$

where $\theta=\left\{\beta_{k}, \Sigma_{k}, \gamma^{r}, \gamma \mid k=0,1\right\}$ represents the model parameters and $m_{i I_{t}}=X_{i t} \beta_{i, I_{t}}$ and $\Sigma_{i j, I_{t}}$ are obtained by gathering the corresponding rows in (1) and by partitioning accordingly the moment matrices. This allows the expression of the joint observation density $f\left(y_{t}^{*}\right)$ as the product of a marginal and a conditional density, $f\left(y_{t}^{*} \mid \cdot\right)=f\left(y_{1 t}^{*} \mid y_{2 t}, \cdot\right) f\left(y_{2 t} \mid \cdot\right)$, where:

$$
\begin{gathered}
f\left(y_{2 t} \mid \cdot\right)=N\left(m_{2 I_{t}}, \Sigma_{22, I_{t}}\right)=N\left(X_{2 t} \beta_{2 I_{t}}, \Sigma_{22, I_{t}}\right) \\
f\left(y_{1 t \mid 2}^{*} \mid \cdot\right)=f\left(y_{1 t}^{*} \mid y_{2 t}, \cdot\right)=N\left(m_{1 I_{t} \mid 2}, M_{1 I_{t} \mid 2}\right)
\end{gathered}
$$

with 


$$
\begin{aligned}
& m_{1 I_{t} \mid 2}=m_{1 I_{t}}+\Sigma_{12, I_{t}} \Sigma_{22, I_{t}}^{-1}\left(y_{2 t}-m_{2 I_{t}}\right) \\
& M_{1 I_{t} \mid 2}=\Sigma_{11, I_{t}}-\Sigma_{12, I_{t}} \Sigma_{22, I_{t}}^{-1} \Sigma_{21, I_{t}}
\end{aligned}
$$

The factoring of $f\left(y_{t}^{*} \mid \cdot\right)$ partitions the joint distribution into two parts and allows the implementation of a normal regression model for the unconstrained variables and a conditional censored normal regression model for the constrained variables

$$
\left[\begin{array}{c}
\tilde{y}_{1|| 2} \\
y_{2 t}
\end{array}\right] \mid X_{t}, I_{t}, \theta \sim N\left(\left[\begin{array}{c}
m_{1 I_{t} \mid 2} \\
m_{2 I_{t}}
\end{array}\right],\left[\begin{array}{cc}
M_{1 I_{t} \mid 2} & 0 \\
0 & \Sigma_{22, I_{t}}
\end{array}\right]\right) 1\left(\tilde{y}_{1 t} \geq b\right)
$$

\section{B Appendix}

\section{Bayesian framework}

\section{B.1 Likelihood}

Define the number $N_{j}, j=1,2$, which indicates the number of, respectively, censored and uncensored variables. Conditional on I and using (19), the data likelihood can be factorized

$$
\begin{gathered}
f(\tilde{\mathbf{y}} \mid \mathbf{X}, \mathbf{I}, \theta)=\prod_{t=p+1}^{T} f\left(\tilde{y}_{t} \mid X_{t}, \beta_{I_{t}}, \Sigma_{I_{t}}\right) 1\left(\tilde{y}_{1 t} \geq b\right) \\
=\prod_{t=p+1}^{T} f\left(\tilde{y}_{1 t} \mid y_{2 t}, X_{1 t}, \beta_{1 I_{t}}, \Sigma_{11, I_{t}}\right) 1\left(\tilde{y}_{1 t} \geq b\right) f\left(y_{2 t} \mid X_{2 t}, \beta_{2 I_{t}}, \Sigma_{22, I_{t}}\right)
\end{gathered}
$$

From (17), the period $t$ density contribution is multivariate normal for $y_{2 t}$

$$
\begin{aligned}
f\left(y_{2 t} \mid X_{2 t}, \beta_{2 I_{t}}, \Sigma_{22, I_{t}}\right) & =(2 \pi)^{-N_{2} / 2}\left|\Sigma_{22, I_{t}}\right|^{-1 / 2} \times \\
& \exp \left\{-\frac{1}{2}\left(y_{2 t}-X_{2 t} \beta_{2 I_{t}}\right)^{\prime} \Sigma_{22, I_{t}}^{-1}\left(y_{2 t}-X_{2 t} \beta_{2 I_{t}}\right)\right\}
\end{aligned}
$$

and the period $t$ contribution of censored variables is

$$
\begin{aligned}
f\left(\tilde{y}_{1 t} \mid y_{2 t}, X_{1 t}, \beta_{1 I_{t}}, \Sigma_{11, I_{t}}\right) 1\left(\tilde{y}_{1 t} \geq b\right)= & \Phi\left(M_{1 I_{t} \mid 2}^{-1 / 2}\left(b-m_{1 I_{t} \mid 2}\right)\right)^{1\left(\tilde{y}_{1 t}=b\right)} \\
& \times\left|M_{1 I_{t} \mid 2}\right|^{-1 / 2} \phi\left(M_{1 I_{t} \mid 2}^{-1 / 2}\left(\tilde{y}_{1 t}-m_{1 I_{t} \mid 2}\right)\right)^{1\left(\tilde{y}_{1 t}>b\right)}
\end{aligned}
$$

where $\Phi\left(M_{1 I_{t} \mid 2}^{-1 / 2}\left(b-m_{1 I_{t} \mid 2}\right)\right)^{1\left(\tilde{y}_{1 t}=b\right)}$ equals

$$
\int_{-\infty}^{b_{N_{1}}} \ldots \int_{-\infty}^{b_{1}}\left|M_{1 I_{t} \mid 2}\right|^{-1 / 2} \phi\left(M_{1 I_{t} \mid 2}^{-1 / 2}\left(\tilde{y}_{1 t}-m_{1 I_{t} \mid 2}\right)\right) d \tilde{y}_{11, t} \ldots d \tilde{y}_{1 N_{1}, t}
$$

and $\phi$ denotes the pdf (see (22)) of the standard (multivariate) normal distribution.

The likelihood of the complete data factorizes

$$
f\left(\boldsymbol{y}^{*} \mid \mathbf{X}, \mathbf{I}, \theta\right)=\prod_{t=p+1}^{T} f\left(y_{1 t}^{*} \mid y_{2 t}, X_{1 t}, \beta_{1 I_{t}}, \Sigma_{11, I_{t}}\right) f\left(y_{2 t} \mid X_{2 t}, \beta_{2 I_{t}}, \Sigma_{22, I_{t}}\right)
$$

where the moments of the marginal and condition normal observation densities are given in, respectively, (17) and (18). 


\section{B.2 Prior distributions}

To complete the Bayesian setup, we specify the prior density of the state indicator I:

$$
\pi\left(\mathbf{I} \mid \mathbf{r}, \gamma, \gamma^{r}\right)=\prod_{t=p+1}^{T} \pi\left(I_{t} \mid r_{t-1}, \gamma, \gamma^{r}\right)
$$

The prior for the censored variables is assumed to be diffuse, $\pi\left(y_{1}^{*}\right) \propto 1\left(y_{1}^{*} \leq b\right)$. We might also work with a proper prior distribution, restricted to the latent area, $\pi\left(y_{1}^{*}\right) \sim N(0, \kappa I) 1\left(y_{1}^{*} \leq b\right)$ with $\kappa$ some real number.

Finally, we assume independent priors for the model parameters:

$$
\pi(\theta)=\pi\left(\gamma, \gamma^{r}\right) \prod_{k=0}^{1} \pi\left(\beta_{k}\right) \pi\left(\Sigma_{k}\right)
$$

The prior for $\left(\gamma^{\mathrm{r}}, \gamma\right)$ includes a state-identifying restriction and additional information on the threshold level, see (10).

The priors on $\beta_{k}$ are independent normal, with variance structure implied by Minnesota priors, $\pi\left(\beta_{k}\right)=$ $N\left(\underline{v}, \underline{V}_{k}\right)$. The vector $\underline{v}$ is of dimension $N(N p+1)$, see (1). Given that we estimate a VAR in levels, we center the first own autoregressive lag at 1 and all other coefficients at zero, $\underline{v}=\left\{\underline{v}_{l} \mid l=1, \ldots, N(N p+1)\right\}$, with

$$
\left(\underline{v}_{l}, \underline{V}_{k, l l}\right)= \begin{cases}1 & l=(j-1)(N p+1)+(j+1), j=1, \ldots, N \\ 0 & \text { otherwise }\end{cases}
$$

We specify the corresponding elements in $\underline{V}_{k}$ as

$$
\operatorname{Var}\left(B_{k l, i j}\right)=\left\{\begin{array}{ll}
0.01 / l^{2} & i=j \\
0.25\left(0.01 / l^{2}\right)\left(\sigma_{k i}^{2} / \sigma_{k j}^{2}\right) & i \neq j
\end{array}, i, j=1, \ldots, N\right.
$$

for $k=0,1$ and $l=1, \ldots, p$. The state-specific variances in the scale factor $\left(\sigma_{k i}^{2} / \sigma_{k j}^{2}\right)$ are equal to the variance of residuals of univariate state-specific autoregressions in which states are predefined as $\underline{I}_{t}=1$ if the libor $\leq 1 \%$. For the intercepts, we work with diffuse priors, $\operatorname{Var}\left(\mu_{k i}\right)=5$.

For $\Sigma_{k}$, we assume an inverse Wishart prior distribution $\operatorname{IW}\left(\underline{s}, \underline{S}_{k}\right)$ with degrees of freedom $\underline{s}=N+2$ and scale $\underline{S}_{k}$ with diagonal elements $\underline{S}_{k, i i}=\sigma_{k i}^{2}$.

\section{B.3 Posterior distributions}

To obtain draws from the posterior

$$
\pi(\vartheta \mid \tilde{\mathbf{y}}) \propto f\left(\mathbf{y}^{*} \mid \mathbf{X}, \mathbf{I}, \theta\right) \pi(\mathbf{I} \mid \mathbf{r}, \theta) \pi\left(\mathbf{y}_{\mathbf{1}}^{*}\right) \pi(\theta)
$$

we sample iteratively from the posterior of

1. the state indicator, $\pi\left(\mathbf{I} \mid \mathbf{y}^{*}, \mathbf{X}, \mathbf{r}, \theta\right)$. Given that there is no state dependence in the state probabilities, we are able to sample the states simultaneously. We update the period $t$ prior odds $P\left(I_{t}=1\right) / P\left(I_{t}=0\right)=$ $\exp \left(\gamma^{r} r_{t-1}+\gamma\right)$ to obtain the posterior odds

$$
P\left(I_{t}=1 \mid \cdot\right) / P\left(I_{t}=0 \mid \cdot\right)=\frac{f\left(y_{t}^{*} \mid X_{t}, \beta_{1}, \Sigma_{1}\right) \exp \left(\gamma^{r} r_{t-1}+\gamma\right)}{f\left(y_{t}^{*} \mid X_{t}, \beta_{0}, \Sigma_{0}\right)}, t=p+1, \ldots, T
$$

We sample $T-p$ uniform random variables $U_{t}$, and set $I_{t}=1$ if

$$
P\left(I_{t}=1 \mid \cdot\right) /\left(P\left(I_{t}=0 \mid \cdot\right)+P\left(I_{t}=1 \mid \cdot\right)\right) \geq U
$$


2. the censored variables, $\pi\left(y_{1}^{*} \mid y_{2}, \mathbf{X}, \mathbf{I}, \theta\right) 1\left(y_{1}^{*} \leq b\right)$. Conditional on $\mathbf{I}$ and the observed variables, and given a diffuse prior, the moments of the posterior normal distribution $\pi\left(\boldsymbol{y}_{1}^{*} \mid \cdot\right)$ are given by (18). We sample from this distribution truncated to the region $y_{1}^{*} \leq b$.

3. the parameters of the state distribution, $\pi(\gamma \mid \mathbf{r}, \mathbf{I}) 1\left(\gamma^{r}<0\right) 1\left(-\gamma^{r} \gamma \leq \gamma \leq-\gamma^{r} \bar{\gamma}\right)$. First, we introduce two layers of data augmentation, which renders the non-linear, non-normal model into a linear-normal model for the parameters (Frühwirth-Schnatter and Frühwirth, 2010):

- We express the state distribution in relative terms, i.e. as difference between the latent state utilities

$$
\begin{aligned}
\omega_{t} & =I_{1 t}^{u}-I_{0 t}^{u} \\
& =\gamma^{r} r_{t-1}+\gamma+\epsilon_{t}, \epsilon_{t} \sim \text { Logistic }
\end{aligned}
$$

where

$$
\begin{aligned}
& I_{1 t}^{u}=\gamma^{r} r_{t-1}+\gamma+v_{1 t} \text {, and } I_{0 t}^{u}=v_{0 t} \\
& \text { with } v_{k t} \text { i.i.d. Type I EV }
\end{aligned}
$$

- We approximate the Logistic distribution by a mixture of normals with $M$ components, $R=\left(R_{1}, \ldots, R_{T}\right)$. Conditional on the latent relative state utilities $\varpi$ and the components, we obtain a normal posterior distribution, $N(g, G)$ with moments:

$$
\begin{aligned}
& G=\left(G_{0}^{-1}+\sum_{t=p+1}^{T} Z_{t}^{\prime} Z_{t} / s_{m_{t}}^{2}\right)^{-1} \\
& g=G\left(G_{0}^{-1} g_{0}+\sum_{t=p+1}^{T} Z_{t}^{\prime} \omega_{t} / s_{m_{t}}^{2}\right)
\end{aligned}
$$

where $Z_{t}=\left[r_{t-1}, 1\right]^{\prime}$ and $s_{m_{t}}^{2}=s_{m}^{2}$ is the variance of the mixture components $R_{t}$, see Table 2 in FrühwirthSchnatter and Frühwirth (2010).

To implement the restrictions on $\gamma$ according to (6), we partition the posterior appropriately:

$$
\pi\left(\gamma^{r}, \gamma \mid \cdot\right) \sim N\left(\left[\begin{array}{l}
g_{1} \\
g_{2}
\end{array}\right],\left[\begin{array}{ll}
G_{11} & G_{12} \\
G_{21} & G_{22}
\end{array}\right]\right)
$$

Then we first sample $\gamma^{r,(m c)}$ from $N\left(g_{1}, G_{11}\right) 1\left(\gamma^{r}<0\right)$ and then sample $\gamma$ from the truncated conditional posterior (Robert, 2009; Botev, 2017):

$$
\gamma \mid \gamma^{r}=\gamma^{r,(m c)} \sim N\left(g_{2}^{c}, G_{2}^{c}\right) 1\left(-\gamma^{r} \underline{\underline{\gamma}} \leq \gamma \leq-\gamma^{r} \bar{\gamma}\right)
$$

with moments

$$
\begin{aligned}
& g_{2}^{c}=g_{2}-G_{21} G_{11}^{-1}\left(\gamma^{r,(m c)}-g_{2}\right) \\
& G_{2}^{c}=G_{22}-G_{21} G_{11}^{-1} G_{12}
\end{aligned}
$$

4. the rest of the parameters, $\pi\left(\theta_{-\gamma} \mid \mathbf{X}, \boldsymbol{y}_{1}^{*}, \mathbf{I}\right)$. Conditional on $\mathbf{I}$ and the augmented data $\boldsymbol{y}^{*}$, the model in (1) is linear. The posterior distribution of the regression parameters and of the error variances are then, respectively, normal and inverse Wishart, the moments of which can be derived in the usual way. 


\section{Appendix}

\section{Addtional results}

\section{C.1 Duration of minimum 2 periods}
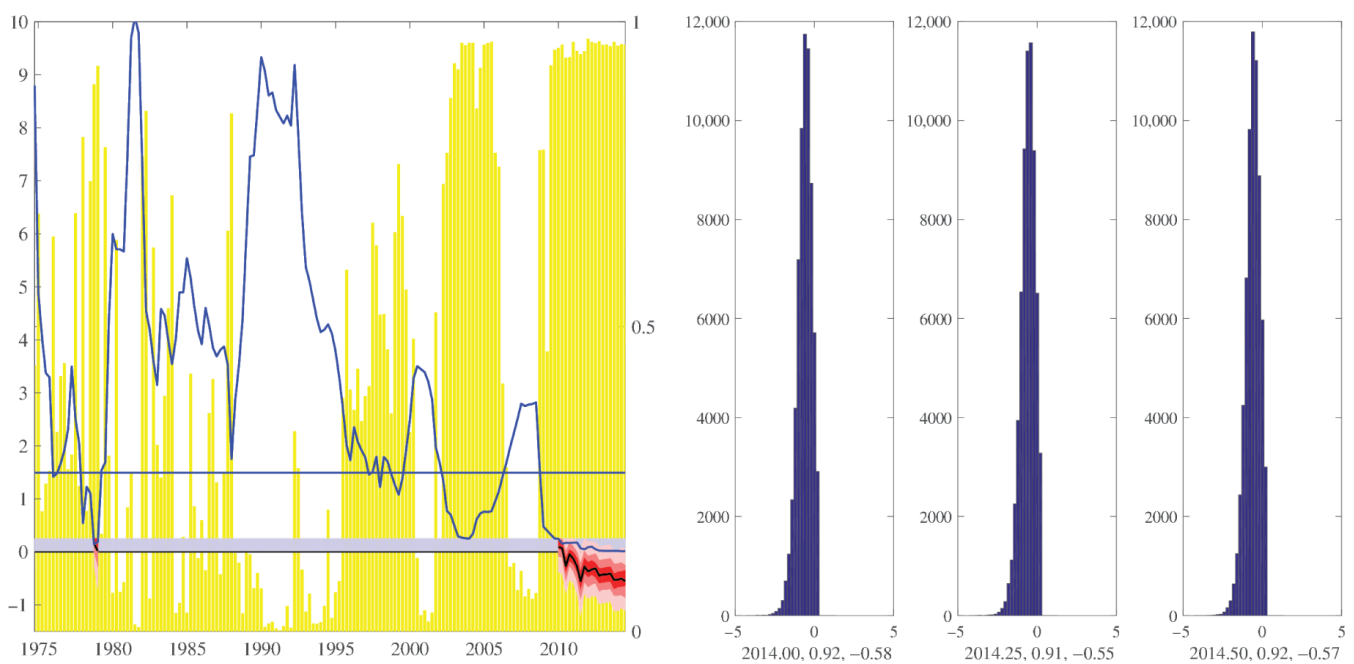

Figure 17: Duration of minimum 2 periods.

Left-hand: Observed interest rate and model-based estimate of $\boldsymbol{y}_{\mathbf{1}}^{*}<b$ (red); the black line is the median, the areas decreasing in shades correspond to, respectively, the $25 \%$, the $50 \%$ and the $80 \%$ interval of highest posterior density; mean posterior probability of state 1 (yellow). Right-hand: histogram of $y_{1 t}^{*}<b$ for $t=2014.00,2014.25,2014.50$, i.e. 2014 first through third quarter; the second and third numbers under each histogram refer to, respectively, $P\left(y_{1 t}^{*}<\min _{t}\left\{y_{1 t}\right\}\right)$ and median $\left(y_{1 t}^{*}\right)$.

\section{C.2 Additional results of the Full model}
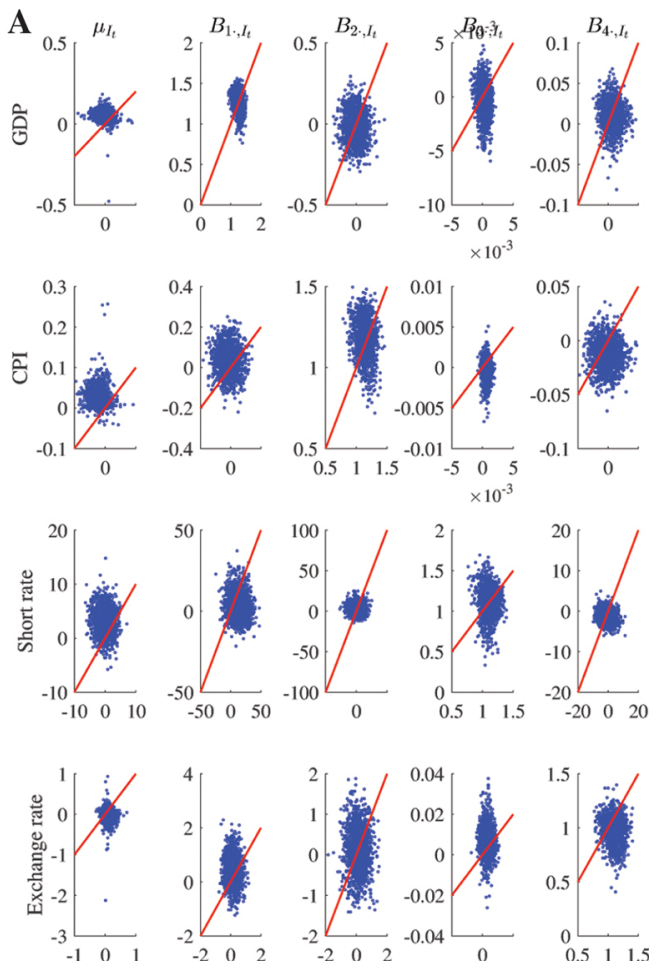

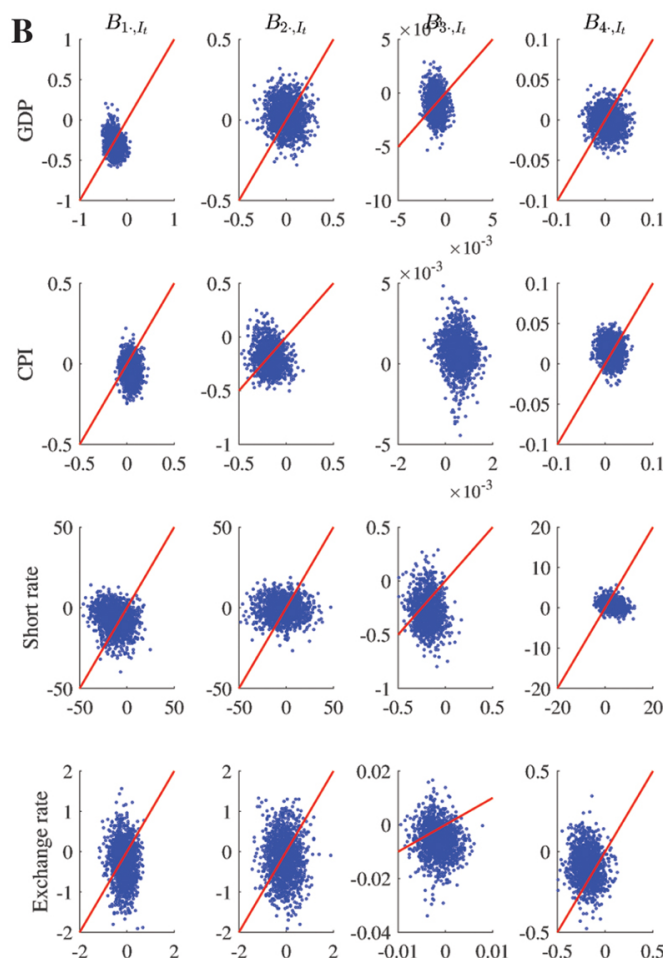

Figure 18: Scatter plots: State $I_{t}=0$ (x-axis) against $I_{t}=1$ (y-axis) parameter draws along with the $45 \%$ line. (A) Intercept and lag 1. (B) lag 2.

\section{C.3 Additional results of the SV model}
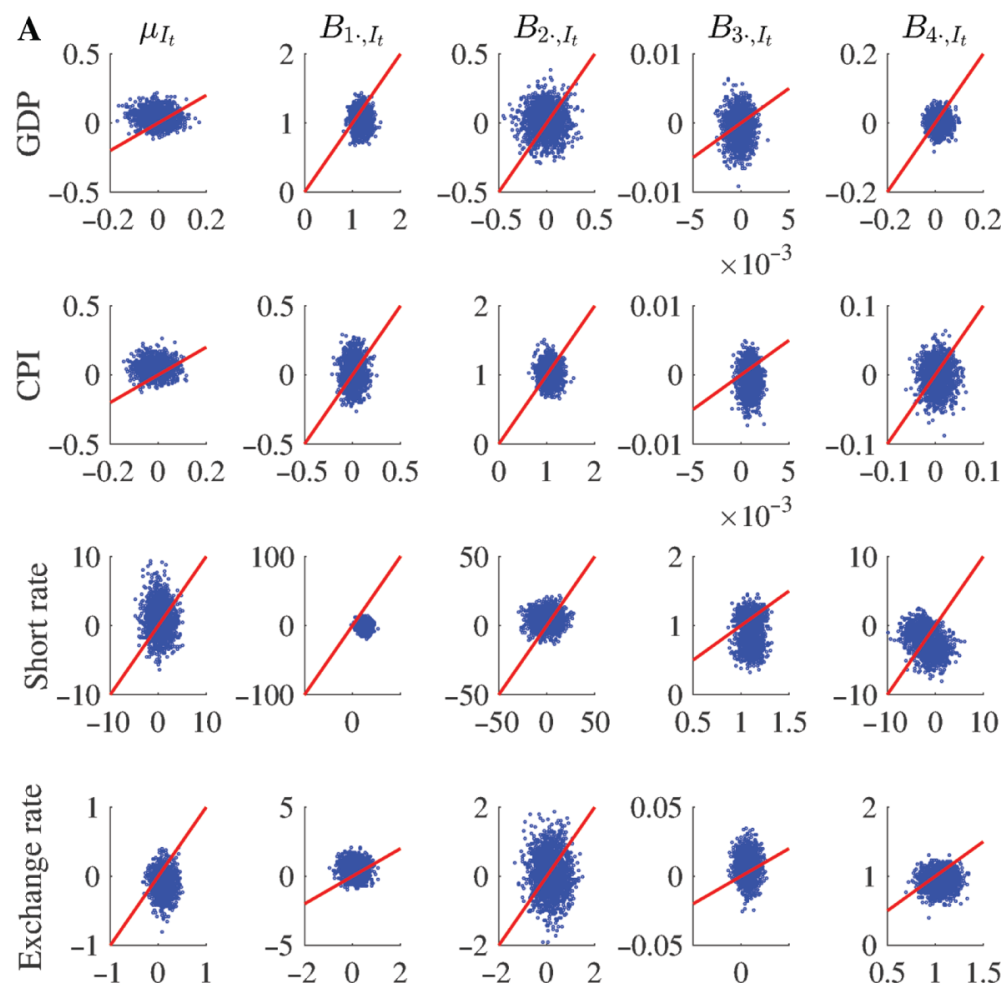

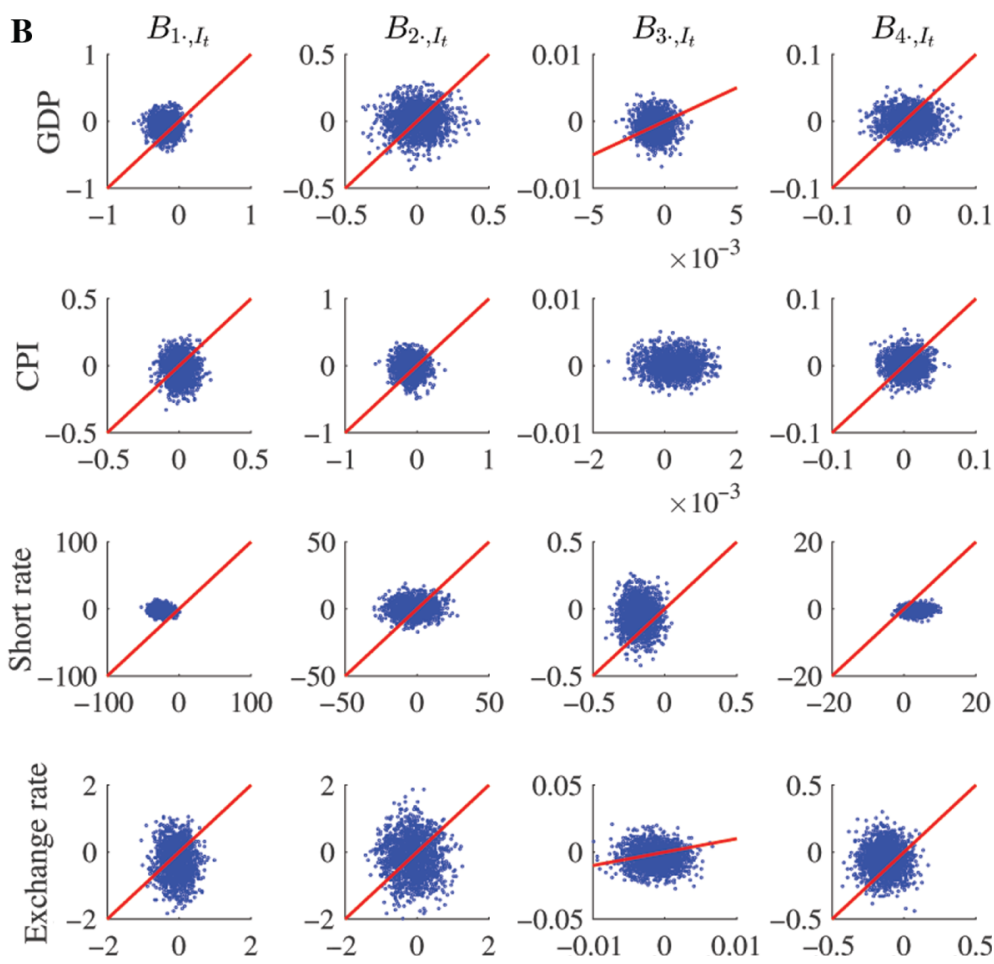

Figure 19: Switching VAR with SV. Scatter plots: State $I_{t}=0$ (x-axis) against $I_{t}=1$ (y-axis) parameter draws along with the $45 \%$ line. (A) Intercept and lag 1. (B) lag 2.

\section{Notes}

1 Additionally, there are a number of contributions focussing on the behaviour of the yield curve at the ZLB (see e.g. Wright, 2012; Christensen and Rudebusch, 2016; Krippner, 2015; Swanson and Williams 2014a; 2014b; Bauer and Rudebusch, 2016).

2 The model can be generalized to $\tilde{y}_{1 t}$ being a sub-vector of $y_{t}$ and $b$ containing different bounds. For example, in the Swiss case, in addition to the interest rate constraint, this could be the 1.20 floor introduced for the euro-Swiss franc exchange rate. Appendices A and B detail, respectively, the distributional properties and the Bayesian framework including sampling steps for a general setup, i.e. for multiple constrained variables.

3 Swanson and Williams (2014b) emphasize, for example, that the effective lower bound is potentially positive for institutional reasons. 4 The results are nearly unchanged if the mean of the interest rate forecast is not allowed to be lower than the lagged forecast, i.e. setting $m_{i T+h}^{(l)} \leq y_{i T+h-1}^{(l)}$ for $h=1, \ldots, H$. The scenario we implement yields smoother interest rate dynamics when exiting the ZLB region.

5 Bäurle and Kaufmann (2018) take into account another brief episode with interest rates as low as $0.5 \%$ in 2003 and 2004, based on the argument that a shadow short-rate fell below the three-month libor and into negative territory.

$6 \mathrm{Wu}$ and Xia (2016) also set $b=0.25$ to estimate a non-linear term-premium model with a shadow short-term policy rate for the US. They justify the threshold by the fact that the lower bound of the target range was 0 by the time the target for the Federal funds rate was lowered to 25 bps.

7 We thank Anne Kathrin Funk for providing the individual participant's interest rate forecasts.

8 Expectations according to futures contracts reflect professonial forecasters' expectations. Before August 2011, market participants expected the libor to remain between 0.2 and $0.25 \%$ during the next quarter.

9 Some of the identified $I_{t}=1$ periods last only one quarter at the beginning of the sample. Enforcing a minimum duration of two quarters leaves posterior inference nearly unchanged, see Figure 17 in online Appendix C.1. Uncertainty about state classification is larger, however. 10 These and the following results are robust to estimating a model in which the upper bound for the threshold is set to $\bar{\gamma}=2.0$. Increasing the censoring value to $b=1.0$ for both specifications of $\bar{\gamma}=\{1.5,2.0\}$ leads to a latent rate that follows closely the observed rate at the end of the 1970s and from mid 2002 to the end of 2005. At the end of the sample the latent rate reaches the zero bound and then passes into negative territory by the third quarter of 2009. Given that, in the current setting, $b=0.25$ and the latent rate turns negative at the beginning of 2010, we can infer that the interest rate was bounded from below at the end of the sample while it was not during the previous periods of low interest rates. These additional findings corroborate our setting $b=0.25$. To save space, the additional results are not reported but available upon request from the authors.

11 For the interested reader, Figure 18 in Appendix C. 2 shows scatter plots of State $I_{t}=0$ (x-axis) against $I_{t}=1$ (y-axis) draws of intercepts and VAR parameters. For some parameters, the posterior distribution is shifted away from the $45 \%$ line. Those are the relevant ones leading to differences in impulse responses.

12 Bäurle and Kaufmann (2018) incorporate the ZLB in a New Keynesian model with Calvo pricing (Galí, 2005). A risk premium shock leads to an immediate and strong drop in the price level when the ZLB is binding, because those firms that adjust reduce strongly their price after the appreciation shock. Results are potentially more in line with our empirical results when strategic complementarity in price setting is present. Firms that adjust like to keep prices close to those of their competitors that do not adjust. Therefore, adjusting firms change prices only by a small amount, which leads to a more gradual but nevertheless protracted decline in the price level.

13 In this basic model with four variables, it is not obvious to identify a monetary policy shock in state $I_{t}=1$ when interest rates are near the ZLB. Therefore, we provide forecasts obtained with the reduced form. 
14 The interested reader finds the derivation of the posterior distributions in Chan and Eisenstat (2018). We thank the authors for sharing their matlab code.

15 Imposing sign restrictions yields 24,292 retained draws for state $I_{t}=0$ and 24,138 retained draws for state $I_{t}=1$. Given that volatilities evolve smoothly, we chose two periods corresponding to $I_{t}=0$ and $I_{t}=1$ in the Full model to draw impulse responses: The first quarter of 1991 and the second quarter of 2005, respectively.

16 The interested reader finds scatter plots of switching parameters in Figure 19 of Appendix C.3

\section{References}

Arias, J. E., J. F. Rubio-Ramírez, and D. F. Waggoner. 2018. “Inference Based on Structural Vector Autoregressions Identified with Sign and Zero Restrictions: Theory and Applications." Econometrica 86: 685-720.

Auerbach, A. J., and M. Obstfeld. 2005. "The Case for Open-Market Purchases in a Liquidity Trap." The American Economic Review 95: 110-137. Auerbach, A. J., and Y. Gorodnichenko. 2012. "Measuring the Output Responses to Fiscal Policy." American Economic Journal: Economic Policy 4: $1-27$.

Bańbura, M., D. Giannone, and L. Reichlin. 2010. “Large Bayesian Vector Auto Regressions.” Journal of Applied Econometrics 25: 71-92.

Bauer, M. D., and G. D. Rudebusch. 2016. "Monetary Policy Expectations at the Zero Lower Bound." Journal of Money, Credit and Banking 48: 1439-1465.

Baumeister, C., and L. Benati. 2013. “Unconventional Monetary Policy and the Great Recession: Estimating the Macroeconomic Effects of a Spread Compression at the Zero Lower Bound." International Journal ofCentral Banking 9: 165-212.

Bäurle, C., and D. Kaufmann. 2018. “Measuring Exchange Rate, Price, and Output Dynamics at the Effective Lower Bound." Oxford Bulletin of Economics and Statistics 80: 1243-1266.

Belmonte, M., G. Koop, and D. Korobilis. 2014. "Hierarchical Shrinkage in Time-Varying Parameter Models." Journal of Forecasting 33: 80-94.

Benhabib, J., S. Schmitt-Grohe, and M. Uribe. 2002. "Avoiding Liquidity Traps." Journal of Political Economy 110: 535-563.

Botev, Z. 2017. "The Normal Law Under Linear Restrictions: Simulation and Estimation via Minimax Tilting." Journal of the Royal Statistical Society, Series B 79: 125-148.

Caggiano, G., E. Castelnuovo, and G. Pellegrino. 2017. “Estimating the Real Effects of Uncertainty Shocks at the Zero Lower Bound.” European Economic Review 100: 257-272.

Chan, J. C. C., and E. Eisenstat. 2018. "Bayesian Model Comparison for Time-Varying Parameter VARs with Stochastic Volatility." Journal of Applied Econometrics 33: 509-532.

Chib, S. 1992. "Bayesian Analysis in the Tobit Censored Regression Model." Journal of Econometrics 51: 79-99.

Christensen, J. H., and C. D. Rudebusch. 2016. “Modeling Yields at the Zero Lower Bound: Are Shadow Rates the Solution?” In Dynamic Factor Models (Advances in Econometrics), edited by E. Hillebrand and S. J. Koopman, Vol. 35, 75-125. Howard House, Wagon Lane, Bingley BD16 1WA, UK: Emerald Croup Publishing Limited.

Clark, T. E. 2011. "Real-Time Density Forecasts from Bayesian Vector Autoregressions with Stochastic Volatility." Journal of Business $\&$ Economic Statistics 29: 327-341.

Debortoli, D., J. Galí, and L. Gambetti. 2018. “On the Empirical (Ir)relevance of the Zero Lower Bound Constraint.” Economics Working Papers 1594, Department of Economics and Business, Universitat Pompeu Fabra.

Diebold, F. X., F. Schorfheide, and M. Shin. 2017. “Real-Time Forecast Evaluation of DSCE Models with Stochastic Volatility." In Theoretical and Financial Econometrics: Essays in Honor ofC. Courieroux, edited by S. Darolles, E. Renault, and A. Monfort, 322-332. Journal of Econometrics 201, Special issue.

Doan, T., R. Litterman, and C. A. Sims. 1984. “Forecasting and Conditional Projection Using Realistic Prior Distributions." Econometric Reviews 3: 1-100.

Eggertsson, G. B., and M. Woodford. 2003. “The Zero Bound on Interest Rates and Optimal Monetary Policy.” Brookings Papers on Economic Activity 34: 139-235.

Eggertsson, G. B., M. Woodford, T. Einarsson, and E. M. Leeper. 2004. “Optimal Monetary and Fiscal Policy in a Liquidity Trap (with Comments)." NBER International Seminar on Macroeconomics 1: 75-144.

Frühwirth-Schnatter, S., and R. Frühwirth. 2010. “Data Augmentation and MCMC for Binary and Multinomial Logit Models." In Statistical Modelling and Regression Structures - Festschrift in Honour of Ludwig Fahrmeir, edited by T. Kneib and C. Tutz, 111-132. Heidelberg: PhysicaVerlag.

Galí, J. and T. Monacelli. 2005. “Monetary Policy and Exchange Rate Volatility in a Small Open Economy.” Review of Economic Studies 72: 707734.

Gust, C. J., J. D. Lopez-Salido, M. E. Smith, and E. Herbst. 2017. "The Empirical Implications of the Interest-Rate Lower Bound." American Economic Review 107: 1971-2006.

Iwata, S., and S. Wu. 2006. “Estimating Monetary Policy Effects when Interest Rates are Close to Zero." Journal of Monetary Economics 53: 13951408.

Jordan, T. 2009. “Die Geldpolitik der Schweizerischen Nationalbank in stürmischen Zeiten.” Speech on 19 March, Swiss National Bank. Kaufmann, S. 2015. "K-state Switching Models with Time-Varying Transition Distributions - Does Loan Growth Signal Stronger Effects of Variables on Inflation?" Journal of Econometrics 187: 82-94.

Kimura, T., and ]. Nakajima. 2016. “Identifying Conventional and Unconventional Monetary Policy Shocks: A Latent Threshold Approach." The B.E. Journal of Macroeconomics 16: 277-300.

Krippner, L. 2015. Zero Lower Bound Term Structure Modeling. New York: Palgrave Macmillan.

Lopes, H. F., and E. Salazar. 2005. “Bayesian Model Uncertainty in Smooth Transition Autoregressions.” Journal of Time Series Analysis 27: 99117. 
Miyao, R. 2002. "The Effects of Monetary Policy in Japan." Journal of Money Credit and Banking 34: 376-392.

Nakajima, J. 2011. “Monetary Policy Transmission Under Zero Interest Rates: An Extended Time-Varying Parameter Vector Autoregression Approach." The B.E. Journal of Macroeconomics 11: 1-24.

Plante, M., A. W. Richter, and N. A. Throckmorton. 2018. "The Zero Lower Bound and Endogenous Uncertainty." The Economic Journal 128: 1730-1757.

Reifschneider, D., and J. C. Williams. 2000. "Three Lessons for Monetary Policy in a Low-Inflation Era." Journal ofMoney, Credit and Banking 32: 936-966.

Robert, C. P. 2009. "Simulation of Truncated Normal Variables." arxiv:0907.4010v1 [stat.co], LSTA, Université Pierre et Marie Curie, Paris.

Schenkelberg, H., and S. Watzka. 2013. "Real Effects of Quantitative Easing at the Zero Lower Bound: Structural VAR-Based Evidence from Japan." Journal of International Money and Finance 33: 327-357.

SNB. 2003. "Swiss National Bank Lowers the Target Range for the Three-Month Libor Rate by 0.5 Percentage Points to o\%-0.75\%." Press release on 6 March, Swiss National Bank.

SNB. 2009. "Swiss National Bank Takes Decisive Action to Forcefully Relax Monetary Conditions." Monetary policy assessment on 12 March, Swiss National Bank.

SNB. 2011. “Die Nationalbank Trifft Massnahmen Gegen den Starken Franken." Press release on 3 August, Swiss National Bank.

Swanson, E. T., and J. C. Williams. 2014a. "Measuring the Effect of the Zero Lower Bound on Medium- and Longer-Term Interest Rates." The American Economic Review 104: 3154-3185.

Swanson, E. T., and J. C. Williams. 2014b. "Measuring the Effect of the Zero Lower Bound on Yields and Exchange Rates in the U.K. and Germany." Journal of International Economics 92: S2-S21.

Teräsvirta, T., and H. M. Anderson. 1992. "Characterizing Nonlinearities in Business Cycles Using Smooth Transition Autoregressive Models." Journal of Applied Econometrics 7: S119-S136.

Woodford, M. 2003. Interest and Prices: Foundations of a Theory of Monetary Policy. Princeton: Princeton University Press.

Wright, J. H. 2012. “What does Monetary Policy do to Long-Term Interest Rates at the Zero Lower Bound?" The Economic Journal 122: F447F466.

Wu, J. S., and F. D. Xia. 2016. "Measuring the Macroeconomic Impact of Monetary Policy at the Zero Lower Bound." Journal of Money Credit and Banking 48: 253-291.

Supplementary Material: The online version of this article offers supplementary material (DOI: https://doi.org/10.1515/snde-2017-0098). 